\title{
TomoPIV meets Compressed Sensing
}

\author{
Stefania Petra and Christoph Schnörr
}

August 9, 2009

\begin{abstract}
We study the discrete tomography problem in Experimental Fluid Dynamics - Tomographic Particle Image Velocimetry (TomoPIV) - from the viewpoint of compressed sensing (CS). The CS theory of recoverability and stability of sparse solutions to underdetermined linear inverse problems has rapidly evolved during the last years. We show that all currently available CS concepts predict an extremely poor worst case performance, and a low expected performance of the TomoPIV measurement system, indicating why low particle densities only are currently used by engineers in practice. Simulations demonstrate however that slight random perturbations of the TomoPIV measurement matrix considerably boost both worst-case and expected reconstruction performance. This finding is interesting for CS theory and for the design of TomoPIV measurement systems in practice.
\end{abstract}

AMS Subject Classifications: 65F22, $68 U 10$

Keywords: compressed sensing, underdetermined systems of linear equations, positivity constraints in ill-posed problems, sparsest solution, TomoPIV

\section{Introduction}

\subsection{TomoPIV}

Our research work is motivated by the work [21]. The authors introduced a new 3D technique, called Tomographic Particle Image Velocimetry (TomoPIV) for imaging turbulent fluids with high speed cameras. The technique is based on the instantaneous reconstructions of particle volume functions from few and simultaneous projections (2D images) of tracer particles within the fluid. The reconstruction of the $3 \mathrm{D}$ image from $2 \mathrm{D}$ images employs a standard algebraic reconstruction algorithm [27].

TomoPIV can use only few projections due to both limited optical access to wind and water tunnels and cost and complexity of the necessary measurement apparatus. As a consequence, the reconstruction problem becomes severely ill-posed, and both the mathematical analysis and the design of algorithms fundamentally differ from the standard scenarios of medical imaging.

A crucial parameter for 3D fluid flow estimation from image measurements is particle density. This parameter also largely influences the tomographical reconstruction problem. Higher 
densities ease subsequent flow estimation and increase the resolution and measurement accuracy. However, higher densities also aggravate ill-posedness of the reconstruction problem. A thorough investigation of this trade-off is lacking. Our objective is to address these this problem taking into account relevant developments in applied mathematics.

TomoPIV adopts a simple discretized model for an image-reconstruction problem known as the algebraic image reconstruction model [1], which assumes that the image consists of an array of unknowns (voxels), and sets up algebraic equations for the unknowns in terms of measured projection data. The latter are the pixel entries in the recorded 2D images that represent the integration of the 3D light intensity distribution $I(z)$ along the pixels line-ofsight $L_{i}$ obtained from a calibration procedure. Thus, the $i$-th measurement obeys

$$
b_{i}: \approx \int_{L_{i}} I(z) d z \approx \sum_{j=1}^{n} x_{j} \int_{L_{i}} \mathcal{B}_{j}(z) d z=\sum_{j=1}^{n} x_{j} a_{i j},
$$

where $a_{i j}$ is the value of the $i$-th pixel if the object to be reconstructed is the $j$-th basis function. The values $a_{i j}$ depend on the choice of the basis function. Typically, $\mathcal{B}_{j}$ are cube-shaped uniform basis functions, the classical voxels. For simplicity we will adopt this discretization scheme and stress that other choices are possible, see e.g. [29].

The main task is to estimate the weights $x_{j}$ from the recorded 2D images, corresponding to basis functions and solve $A x \approx b$. The matrix $A$ has dimensions (\# pixel $=: m$ ) $\times$ (\# basis functions $=n$ ), where $m \ll n$. Since each row indicates those basis functions whose support intersect with the corresponding projection ray the projection matrix $A$ will be sparse.

\subsection{Compressed Sensing}

We study the tomographic problem of reconstructing particle volume functions from the general viewpoint of Compressed Sensing, which is a central theme of current research in applied mathematics. Compressed Sensing $[10,11,17]$ is a new technique for acquiring a sparse signal $x^{*} \in \mathbb{R}^{n}$ by incomplete linear measurement

$$
A x=b,
$$

where $A \in \mathbb{R}^{m \times n}, m<n$, and for reconstructing $x^{*}$ exactly provided that the signal is sparse (or compressible in some basis), i.e. $\left\|x^{*}\right\|_{0}:=\left|\left\{i \mid x_{i}^{*} \neq 0\right\}\right| \ll n$.

Instead of considering the NP-hard $\ell_{0}$-minimization problem

$$
\min \|x\|_{0} \quad \text { s.t. } A x=b \text {, }
$$

it considers the convex $\ell_{1}$-minimization problem

$$
\min \|x\|_{1} \quad \text { s.t. } A x=b \text {, }
$$

and investigates the situations when the same $x^{*}$ solve both problems (2) and (3), coined as $\ell_{0} / \ell_{1}$-equivalence.

A remarkable result of Candès and Tao [11] is that if, for example, the rows of $A$ are randomly chosen Gaussian distributed vectors, there is a constant $C$ such that if the signal 
sparsity level obeys $\left\|x^{*}\right\|_{0}<C m / \log \left(\frac{n}{m}\right)$, the solution of (3) will be exactly the original signal $x^{*}$ with overwhelming probability.

In fact, random measurement matrices are optimal $[9,19,28,3]$ in the sense that for a given sparsity level $k$, the required number of samples is minimal such that $\ell_{0} / \ell_{1}$-equivalence holds. On the other hand, for a given number of measurements $m$ the sparsity level $k$ of $x^{*}$ which allows recovery by $\ell_{1}$-minimization is maximal. The different derivations of $\ell_{0} / \ell_{1}$ equivalence are quite involved and are based on the notion of Restricted Isometry Property $(R I P)$ [3], see Section 4.3, or on "counting faces" of polytops $[15,19,16]$.

When the solution is known to be sparse and positive then under a similar assumption on $A, k$ and $m$ all nonnegative $k$-sparse vectors $x^{*}$ are the unique positive solution of $A x=A x^{*}$, $[7,19]$.

Donoho and Tanner $[16,19]$ have computed sharp reconstruction thresholds for Gaussian measurements, such that for any choice of sparsity $k$ and signal size $n$, the required number of measurements $m$ to recover $x^{*}$ can be determined precisely.

Recent trends $[4,5,24]$ tend to replace random dense matrices by adjacency matrices of "high quality" unbalanced expander graphs. Here, the measurement matrices $A$ are sparse binary matrices.

\subsection{Stylized Problem}

Likewise, we will concentrate on a particular binary measurement matrix. We consider a 3D image $I$ with a cubic domain $V$ discretized in $d^{3}$ voxels. Three cameras, with $d^{2}$ pixels $\left(L_{i}\right.$ rays) each, keep the volume under investigation in focus from three orthogonal directions, compare Fig. 1.3 (left). According to Section 1.1 each entry of the measurement matrix $A$ will be

$$
a_{i j}=\int_{L_{i}} \mathcal{B}_{j}(x) d z=1
$$

if the line of sight $L_{i}$ of the $i$-th pixel intersects the $j$-th voxel $\mathcal{B}_{j}$, or $a_{i j}=0$ if not. By numbering voxels and pixels according to Fig. 1.3 (left) matrix $A$ can be written in closed form as

$$
A=\left(\begin{array}{c}
\mathbf{1}_{d}^{\top} \otimes I_{d} \otimes I_{d} \\
I_{d} \otimes \mathbf{1}_{d}^{\top} \otimes I_{d} \\
I_{d} \otimes I_{d} \otimes \mathbf{1}_{d}^{\top}
\end{array}\right),
$$

where $\otimes$ denotes the Kronecker product, see [22]. Notice that $A$ is the adjacency matrix of a bipartite graph with regular left degree 3 and regular right degree $d$, compare Fig. 1.3 (right). The left variable nodes of which there are $n:=d^{3}$ correspond to the voxels in the $d^{3}$ cube and thus to the entries of $x$. The right nodes (or measurement nodes) of which there are $m:=3 d^{2}$ correspond to the camera pixel. In a bipartite graph connections within the variable nodes and within the right nodes do not occur. The existing edges between the left nodes and right nodes are represented by our $m \times n$ matrix $A$ from (4). In particular,

$$
a_{i j}= \begin{cases}1, & \text { if } j \text {-th ray intersects } i \text {-th voxel, } \\ 0, & \text { otherwise }\end{cases}
$$

for all $i \in\{1, \ldots, n\}, j \in\{1, \ldots, m\}$. 

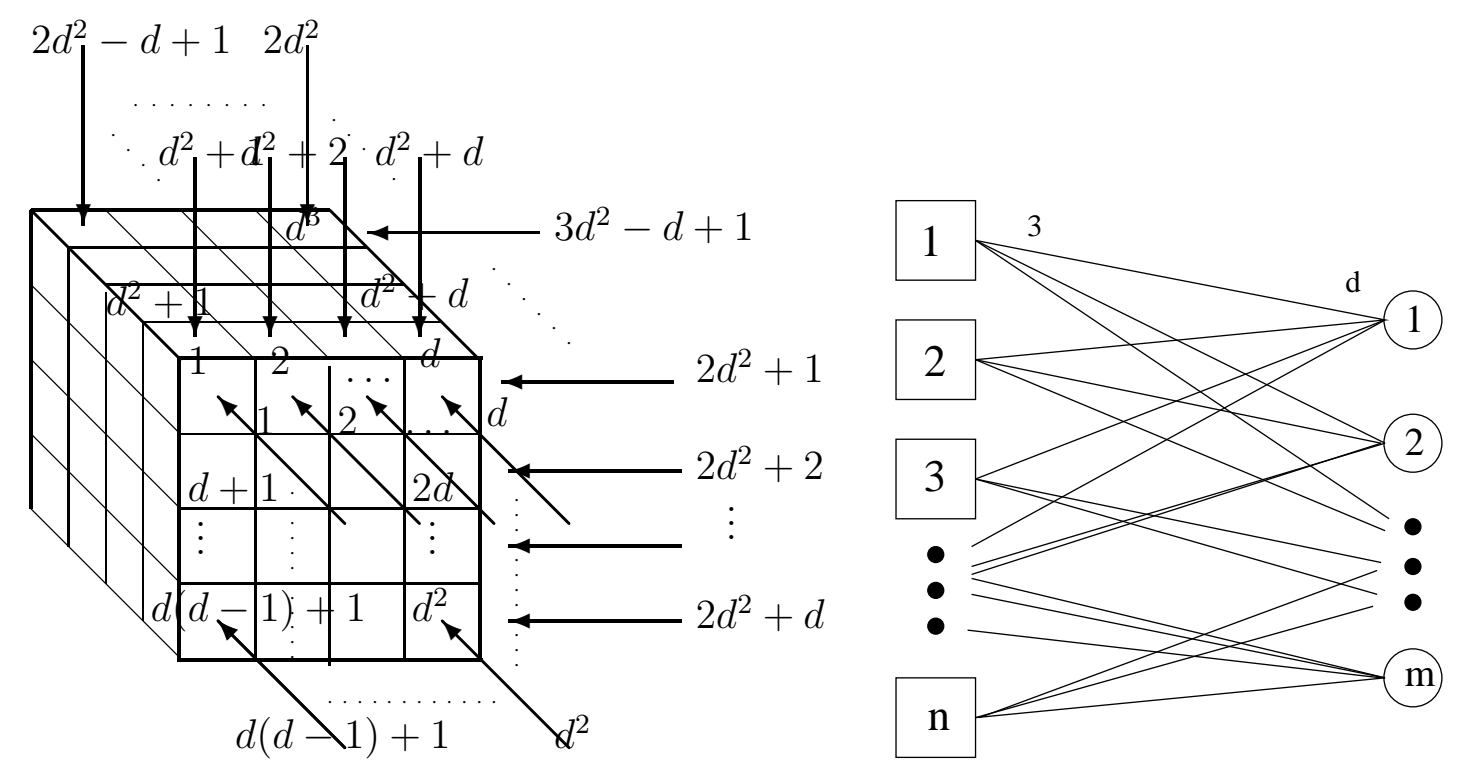

Figure 1: Left: Discretization of the $d \times d \times d$ volume and corresponding $3 d^{2}$ rays for the 3 orthogonal projections. Right: $A$ is the adjacency matrix of a bipartite graph with regular left degree 3 and regular right degree $d$.

Throughout this paper we denote by $x^{*}$ the indicator vector corresponding to original the particle distribution and assume that our measurements based on the sampling matrix $A$ are exact, i.e. $b=A x^{*}$. Moreover, we assume that $x^{*}$ is positive and sparse.

We investigate the sparsity level of $x^{*}$ up to which the the sparsest solution of $A x=A x^{*}$ is unique. Furthermore, we are interested in recovering $x^{*}$ as minimizer of the $\ell_{1}$-minimization problem (3) or, as minimizer of the linear program

$$
\min \mathbf{1}^{\top} x, \quad A x=b, x \geq 0 .
$$

\subsection{Contribution and Organization}

We provide a detailed study of the TomoPIV problem from the viewpoint of compressed sensing. We assess the worst-case and average performance of this severely ill-posed reconstruction problem of discrete tomography, based on convex $\ell_{1}$-regularization and on a range of recently established theoretical results.

The critical parameter both in theory and in practice is the particle density of the imaged fluid, that in mathematical terms corresponds to the sparsity of the vector to be reconstructed from observed measurements. Of particular interest are phase transitions of this parameter below of which unique reconstructions can be assumed to hold in practice - an essential requirement for subsequent processing steps for, e.g., estimating fluid flow velocity from a sequence of reconstructed volume functions. On the other hand, using as large as possible particle densities is important in practice too, in order to improve the spatio-temporal resolution of observed fluid structures.

After establishing basic properties of the measurement matrix (4) in Section 2, we clarify in Sections 3 and 4 the relationship between the regularized reconstruction problems (2), 
(3) and (5) and assess the worst-case and average performance by applying recently established results from the theory of compressed sensing to the TomoPIV problem. Taking into account that sparse volume functions generate sparse observations, we provide in Section 5 a probabilistic analysis of TomoPIV reconstructions based on systems (1) that have been reduced accordingly in a preprocessing step. Finally, we discuss in Section 6 the statistics of numerical simulations based on slightly and randomly perturbed measurement matrices $A$.

In a nutshell, we show that the TomoPIV problem is quite degenerate from the viewpoint of compressed sensing, thus leading to poor performance guarantees (Sections 3,4). On the other hand, the probabilistic analysis of Section 5 yields average performance bounds that back up current rules of thumb of engineers for choosing particle densities in practice. Finally, Section 6 indicates a dramatic performance boost based on only slightly modified measurement systems, raising novel problems for theory and implications for the improved design of real TomoPIV measurement systems.

While Section 3 is based on established theoretical concepts, all remaining sections - and Section 3 too - contain novel material from the specific viewpoint of TomoPIV and also from the more general viewpoint of discrete tomography. In particular, our papers aims at pointing out connections between the fields of compressed sensing and discrete tomography in order to stimulate further research.

\subsection{Notation}

$|X|$ denotes the cardinality of a finite set $X$. We already introduced the pseudo-norm $\|x\|_{0}=$ $\left|\left\{i \mid x_{i} \neq 0\right\}\right|$ and denote the set of $k$-sparse vectors by $\mathbb{R}_{k}^{n}=\left\{x \in \mathbb{R}^{n} \mid\|x\|_{0} \leq k\right\}$. The support of a vector $x \in \mathbb{R}^{n}, \operatorname{supp}(x) \subseteq\{1,2, \ldots, n\}$, denotes the set of indices of nonvanishing components of $x$. With $I^{+}(x)=\left\{i \mid x_{i}>0\right\}, I^{0}(x)=\left\{i \mid x_{i}=0\right\}$ and $I^{-}(x)=\left\{i \mid x_{i}<0\right\}$, we have $\operatorname{supp}(x)=I^{+}(x) \cup I^{-}(x)$ and $\|x\|_{0}=|\operatorname{supp}(x)|$.

If $S$ denotes a finite set then $\mathcal{N}(S)$ denotes the union of all neighbors of elements of $S$, where the corresponding relation (graph) should be clear from the context.

$A_{\bullet}, i$ denotes the $i$-th column vector of a matrix $A$. For given index sets $I, J$, matrix $A_{I J}$ denotes the submatrix of $A$ with rows and columns indexed by $I$ and $J$, respectively. $I^{c}, J^{c}$ denote the respective complement sets. Similarly, $b_{I}$ denotes a subvector of $b$.

$\mathbb{E}[\cdot]$ denotes the expectation operation applied to a random variable.

\section{Preliminaries}

The objective of this section is an examination of the properties of the system (1) for this simple prototype of data-collection geometry. Such properties will be also relevant for other regular imaging geometries, e.g. when additionaly using a fourth camera (projection direction).

By the nature of the problem the coefficient matrix $A$ is very sparse, in contrast to most compressed sensing measurement ensembles. This together with the sparsity of the original signal $x^{*}$ induces a sparsity also in the measurement vector $b$ which in more classic scenarios is not given. As a consequence, we can remove equations with zero right-hand side leading us to a feasible set of reduced dimensionality as will be detailed next. 
Consider the feasible polyhedral set with respect to $A$ and $b$

$$
\mathcal{F}:=\{x \mid A x=b, x \geq 0\},
$$

where all entries $a_{i j}$ in $A$ are nonnegative. Let us introduce the following partitions of the right and left nodes

$$
\begin{aligned}
& I:=I^{0}(b)=\left\{i \in\{1, \ldots, m\} \mid b_{i}=0\right\} \quad \text { and } \quad I^{c}, \\
& J:=\mathcal{N}(I)=\left\{j \in\{1, \ldots, n\} \mid \exists i \in I: a_{i j}>0\right\} \quad \text { and } \quad J^{c} .
\end{aligned}
$$

Further define

$$
\mathcal{F}_{\text {red }}:=\left\{x \mid A_{I^{c} J^{c}} x=b_{I^{c}}, x \geq 0\right\} .
$$

Then we can make the simple, compare [29, Prop.1], but important observation.

Proposition 2.1. Let $A \in \mathbb{R}^{m \times n}, b \in \mathbb{R}^{m}$ have all nonnegative entries and $\mathcal{F}$ and $\mathcal{F}_{\text {red }}$ defined as in (6) and (7) respectively. Then

$$
\mathcal{F}=\left\{x \in \mathbb{R}^{n} \mid x_{J}=0 \text { and } x_{J^{c}} \in \mathcal{F}_{\text {red }}\right\} .
$$

Remark 2.1. Assume that for a particular measurement vector $b$, which induces the partitions $I, I^{c}$ and $J, J^{c}$ of the right and left nodes as defined above, we obtained an overdetermined and full rank submatrix $A_{I^{c} J^{c}}$. Then the vector $x_{J^{c}}^{*}$ is the unique solution of $A_{I^{c} J^{c}} x=b_{I^{c}}$ and $x^{*} \in \mathbb{R}^{n}$, where $x_{J}^{*}=0$, is the the unique positive solution of $A x=b$.

Clearly, when the above situation occurs solving the $\ell_{0}$-problem (2) amounts to solve a feasibility problem. Moreover, any method which solves

$$
\min _{x \in \mathcal{F}} f(x)
$$

for an arbitrary objective function $f$ will lead to the same correct result.

Let us assume for the time being that we have a sufficiently sparse vector $x^{*}$ and a sufficiently sparse measurement vector $b=A x^{*}$ such that $A_{I^{c} J^{c}}$ is overdetermined, i.e.

$$
\left|\mathcal{N}\left(I^{0}(b)\right)\right|-\left|I^{0}(b)\right| \geq n-m .
$$

The rank of $A_{I^{c} J^{c}}$ will equal the rank of $A_{J^{c}}$, while the latter cannot be full if it contains a subset of linearly dependent columns.

This observation motivates us to find an upper bound to the maximal number $s$ of columns such that all $s$ (or less) column combinations are linearly independent. An useful tool for achieving this task, which is in general of combinatorial nature, is to investigate the nullspace of $A$.

Proposition 2.2. Let $d \in \mathbb{N}, d \geq 3$, A from (4) and $N \in \mathbb{R}^{d^{3} \times(d-1)^{3}}$ defined as

$$
N:=\left(\begin{array}{c}
-\mathbf{1}_{d-1}^{\top} \\
I_{d-1}
\end{array}\right) \otimes\left(\begin{array}{c}
-\mathbf{1}_{d-1}^{\top} \\
I_{d-1}
\end{array}\right) \otimes\left(\begin{array}{c}
-\mathbf{1}_{d-1}^{\top} \\
I_{d-1}
\end{array}\right)
$$

Then the following statements hold 
$A$

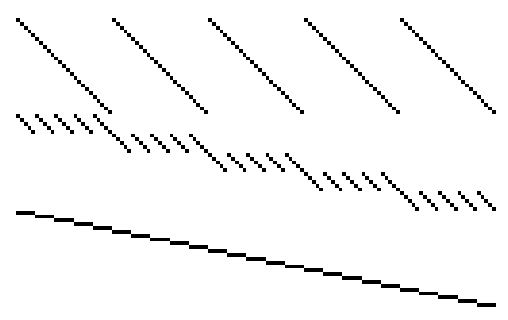

basis for $\operatorname{ker}(A)$

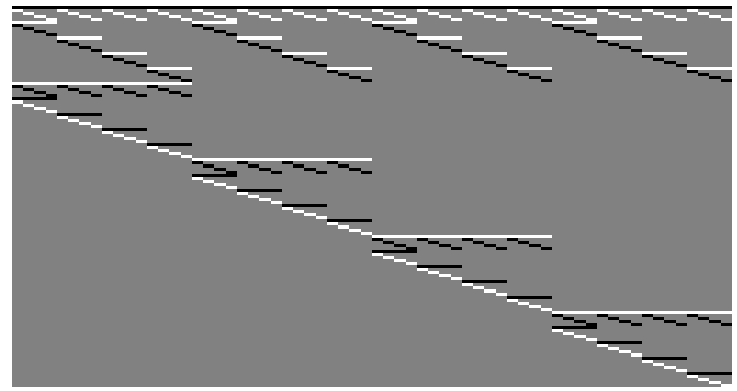

Figure 2: Matrix $A$ from (4) for $d=5$ (left) along with a sparse basis for its nullspace, the columns of $N$ from (9) (right).

(a) $A N=0$, with A from (4).

(b) Every column in $N$ has exactly 8 nonzero elements.

(c) $N$ is a full rank matrix and $\operatorname{rank}(N)=(d-1)^{3}$.

(d) $\operatorname{rank}(A)=3 d^{2}-3 d+1$.

(e) $\operatorname{ker}(A)=\operatorname{span}\{N\}$, i.e. the columns of $N$ provide a basis for the nullspace of $A$.

(f) $\sum_{i=1}^{n} v_{i}=0$ holds for all $v \in \operatorname{ker}(A)$.

Proof. See appendix.

\section{Unique Sparsest Solution}

In order to study $\ell_{0} / \ell_{1}$-equivalence for $A$ from (4) we decompose this problem in two separate conditions:

1. $\ell_{0}$-unique-optimality: $x^{*}$ is the unique optimum of (2);

2. $\ell_{1}$-unique-optimality: $x^{*}$ is the unique optimum of (3).

In this section we investigate the first subproblem, while the second one will be addressed in the next section.

\subsection{Spark}

Besides being one of the classical NP-hard problems, see [25] for this NP-hardness result, problem (2) has a highly nonconvex objective function and thus many local optima may occur. Fortunately previous work has shown that if a sparse enough solution to (2) exists than it will be necessarily unique. The analysis in [20] involves the measure $\operatorname{spark}(A)$ which equals the 
minimal number of linearly dependent columns of $A$, see $[18,20]$. In contrast to $\operatorname{rank}(A)$, $\operatorname{spark}(A)$ is NP-hard to compute. Fortunately bounds on this measures can be derived, [18] and Section 4.2.

The following result is surprisingly elementary and can be found in [18].

Theorem 3.1. (Uniqueness) Let $x^{*}$ be a solution of (1) with $\left\|x^{*}\right\|_{0}<\frac{\operatorname{spark}(A)}{2}$. Then $x^{*}$ is the unique solution of (2).

Clearly, $2 \leq \operatorname{spark}(A) \leq \operatorname{rank}(A)+1$. Again, Gaussian matrices $A \in \mathbb{R}^{m \times n}, m<n$, are optimal in the sense that $\operatorname{spark}(A)$ is maximal and equals $\operatorname{rank}(A)+1=m+1$. Unfortunately, with $A$ from (4) we come off badly.

Proposition 3.2. For all $d \in \mathbb{N}, d \geq 3$ the minimal number of linearly dependent columns of matrix A from (4) equals 8, i.e. $\operatorname{spark}(A)=8$.

Proof. See appendix.

Hence, Thm. 3.1 and Prop. 3.2 yield guaranteed uniqueness of every 3-sparse vector $x^{*}$ only. This bound is tight, since we can construct two 4-sparse solutions $x^{1}$ and $x^{2}$ such that $A x^{1}=A x^{2}$, compare Fig. 3 .

\subsection{Signature}

In [20] Elad adopts a probabilistic point of view to study uniqueness of sparse solutions of (2) beyond the worst-case scenario based on the signature of a matrix $A \in \mathbb{R}^{m \times n}$. This is defined as the discrete function $\operatorname{sig}_{A}(k) \in[0,1]$, for $k \in\{2, \ldots, n\}$, that equals the number of $k$ column combinations in $A$ which are linearly dependent divided by the number of all $k$ columns from the $n$ existing ones. By definition $\operatorname{sig}_{A}(k)=0$, for all $k<\operatorname{spark}(A)$.

Theorem 3.3. [20, Th. 6,Th. 5] Let $\sigma:=\operatorname{spark}(A) \leq \operatorname{rank}(A)=: r$ and $x^{*}$ be a solution $A x=b$. Assume the locations of the nonzero entries in $x^{*}$ are chosen at random with equal and independent probability. If $1 / 2 \sigma \leq\left\|x^{*}\right\|_{0}=: k \leq r$, then the probability that $x^{*}$ is the sparsest solution of $A x=b$ is $1-\operatorname{sig}_{A}(k)$ and the probability to find a solution of $A x=b$ of the same cardinality $k$ is

(a) $\sum_{j=0}^{k-\sigma}(k-j)(n-k+j)\left(\begin{array}{c}k \\ j\end{array}\right) \operatorname{sig}_{A}(k-j)$ or lower, if $\left\|x^{*}\right\|_{0} \geq \sigma$;

(b) 0 , if $1 / 2 \sigma \leq\left\|x^{*}\right\|_{0}<\sigma$.

Hence uniqueness of the sparsest solution with cardinality less then $\operatorname{spark}(A)$ can be claimed with probability 1 .

An upper bound on the signature was derived via arguments from matroid theory [6], under the assumption that the spark is known.

Theorem 3.4. [20, Th. 7] Let $A \in \mathbb{R}^{m \times n}$ with the signature function $\operatorname{sig}_{A}$, $\operatorname{spark}(A)=\sigma$ and $\operatorname{rank}(A)=r$. Then

$$
\operatorname{sig}_{A}(k) \leq 1-\frac{\sum_{i=1}^{\sigma-1}\left(\begin{array}{c}
n-r+i-1 \\
i
\end{array}\right)\left(\begin{array}{c}
r-i \\
k-i
\end{array}\right)}{\left(\begin{array}{l}
n \\
k
\end{array}\right)}, \quad 0 \leq k \leq r .
$$


To compute the signature seems even harder then computing the spark. However, the signature will be close to zero for $k$ small enough, but growing with the dimension of $A$. If $\operatorname{spark}(A)=8$ it does not necessarily mean that every $k$ column combination are linearly dependent. In fact, only a limited number of $k$ column combinations can be dependent without violating $\operatorname{rank}(A)=3 d^{2}-3 d+1$. It turns out that this number is tiny for smaller $k$ when compared to $\left(\begin{array}{l}n \\ k\end{array}\right)$. As $k$ increases this number also grows and equals one only when $k>r$, compare Fig. 6.1 and Fig. 7 (left). Numerical experiments suggest that most $0.9 d^{2}$ column combinations in $A$ are linearly independent.

\section{Unique Positive Solution}

This section might seem useless from a practical point of view since $\ell_{0} / \ell_{1}$-equivalence cannot be claimed for all $k$-sparse vectors when $k$ exceeds 3 in view of the nonuniqueness of the $\ell_{0}$-minimizer in this case. However, we trace relevant conditions yielding $\ell_{0} / \ell_{1}$-equivalence, review known connections between different concepts and attempt to establish some new ones.

\subsection{Relations between problems (3) and (5)}

Most Compressed Sensing results explore conditions under which the minimum of the $\ell_{1}$ minimization problem (3) is unique (and identical to the $\ell_{0}$-minimization problem (2)). We note in this section that if the measurement matrix $A$ has equal column sum and if a positive solution $x^{*}$ to $A x=b$ exists, then a unique $\ell_{1}$-minimizer must equal $x^{*}$. Conversely, if the solution of (5) $x^{*}$ is unique then also the $\ell_{1}$-minimizer must be unique.

Proposition 4.1. If $x^{*}$ solves $A x=b$ with Afrom (4) whereas $\mathbf{1}^{\top} x^{*}=: c$ then for all solutions $x$ of (1) the entries sum equals $c$, i.e. $\mathbf{1}^{\top} x=c$.

Proof. Follows directly from Prop. 2.2 (f).

Lemma 4.2. Assume there is a positive solution $x^{*}$ to $A x=b$ with Afrom (4). Then $x^{*}$ solves the $\ell_{1}$-minimization problem (3).

Proof. Denote by $x^{1}$ a solution to the $\ell_{1}$-minimization problem (3). In view of Prop. 4.1 we obtain

$$
\mathbf{1}^{\top} x^{*}=\mathbf{1}^{\top} x^{1} \leq\left\|x^{1}\right\|_{1} \leq \mathbf{1}^{\top} x^{*},
$$

where the last inequality holds since $x^{*}$ is feasible. Thus equality must hold.

This immediately implies

Proposition 4.3. Assume there is a positive solution $x^{*}$ to $A x=b$, with A from (4) and let $x^{1}$ be the unique solution of the $\ell_{1}$-minimization problem (3). Then $x^{1}$ must equal $x^{*}$.

On the other hand, we have

Proposition 4.4. Consider A from (4) and assume that the positive solution $x^{*}$ to $A x=b$ is unique. Then $x^{*}$ will be also the unique minimizer of the $\ell_{1}$-minimization problem (3). 
Proof. Note that if $x^{*}$ is the unique minimizer of (5) then $x^{*}$ is necessarily $k$-sparse for some $k<n$. Otherwise, it cannot be unique since for $x^{*}$ with no vanishing entries $x^{*}+t v$ will also solve (5) for $t$ sufficiently small and $v \in \operatorname{ker}(A)$. Hence $S:=I^{0}\left(x^{*}\right) \neq \emptyset$. Moreover, $S \cap I^{-}(v) \neq \emptyset$ or $S^{c} \cap I^{-}(v) \neq \emptyset$ hold for all $v \in \operatorname{ker}(A) \backslash\{0\}$, in view of the uniqueness of $x^{*}$. From $\sum_{i \in S} v_{i}+\sum_{i \in S^{c}} v_{i}=0$ we now obtain

$$
\left|\sum_{i \in S^{c}} \operatorname{sign}\left(x_{i}^{*}\right) v_{i}\right|=\left|\sum_{i \in S^{c}} v_{i}\right|<\sum_{i \in S}\left|v_{i}\right|
$$

for all $v \in \operatorname{ker}(A) \backslash\{0\}$. This is a well known condition for the uniqueness of the $\ell_{1}$-minimizer, see e.g. [26].

Note, that the above results hold for all matrices $A$ with equal column sum.

\subsection{Mutual incoherence}

The mutual coherence of a matrix $A$, denoted by $\mu(A)$, is defined as the maximal absolute scalar product between two different normalized columns of $A$,

$$
\mu(A)=\max _{\substack{i, j \\ i \neq j}} \frac{\left\langle A_{\bullet, i}, A_{\bullet, j}\right\rangle}{\left\|A_{\bullet, i}\right\|\left\|A_{\bullet, j}\right\|}
$$

It measures the similarity between the matrix's columns. For an orthogonal matrix $A, \mu(A)=$ 0 . For an $m<n$ we necessarily have $\mu(A)>0$. Uniqueness of the sparsest solution and exact recovery of $x^{*}$ via $\ell_{1}$-minimization can be guaranteed [18] if

$$
\left\|x^{*}\right\|_{0} \leq 0.5\left(1+\frac{1}{\mu(A)}\right)
$$

Hence, there is an interest in matrices with as small as possible $\mu(A) \cdot \mu(A)=1$ implies the existence of two "parallel" columns, and this causes confusion in the construction of a sparse representation of the measurement vector $b$. In [30] it was shown that for a full rank matrix of size $m \times n$

$$
\mu(A) \geq \sqrt{\frac{n-m}{m(n-1)}}
$$

and equality is obtained for a family of matrices called Grassmanian frames.

The mutual coherence is often use to lower bound the spark, since the following relationship always holds

$$
\operatorname{spark}(A) \geq 1+\frac{1}{\mu(A)}
$$

In [7], nonnegativity is taken into account. Here a one-sided coherence is considered

$$
\nu(A)=\max _{\substack{i, j \\ i \neq j}} \frac{\left\langle A_{\bullet, i}, A_{\bullet}\right\rangle}{\left\|A_{\bullet, i}\right\|^{2}} .
$$

The authors obtained the following result. 
Theorem 4.5. [7, Thm. 2] Let $A \in \mathbb{R}^{m \times n}$ be a matrix with nonnegative entries such that all solutions of $A x=b$ satisfy $1^{\top} x=c$, where $c$ is some constant. If there is a nonnegative sparse solution $x^{*}$ to this system with $\left\|x^{*}\right\|_{0}<0.5\left(1+\frac{1}{\nu(A)}\right)$, then it is a unique solution of this problem.

For our particular matrix $A$ we obtain

Proposition 4.6. For all $d \in \mathbb{N}, d \geq 3$ and $A$ defined in (4)

$$
\mu(A)=\nu(A)=\frac{1}{3} .
$$

Proof. Since every column contains exactly 3 ones we obtain $\left\|A_{\bullet}, i\right\|^{2}=3$ for all $i \in\{1, \ldots, n\}$. Thus $\mu(A)=\nu(A)$. Since two different voxels can both be intersected by at most one ray the maximal common support of two different columns is one. This immediately implies the result.

However, recovery bounds based on this bound are too pessimistic since, due to the above result we obtain guaranteed recovery via $\ell_{1}$-minimization for all $k$-sparse vectors if $k<2$, which is (needless to say) useless. Derivation of stronger results that refer to specific matrices and bypass the use of the mutual coherence should be attempted.

\subsection{Restricted Isometry Property}

In order to prove that there exist matrices $A$ with only $m=O(k \log (n / k))$ rows such that for all $k$-sparse $x \ell_{0} / \ell_{1}$-equivalence holds Candès and Tao [10] introduced a concept that outranks the coherence measure $\mu(A)$. A matrix A is said to have the Restricted Isometry Property $R I P_{2, \delta, k}$ if for any $k$-sparse vector $x$, the following expression is verified

$$
(1-\delta)\|x\|^{2} \leq\|A x\|^{2} \leq(1+\delta)\|x\|^{2}, \quad \delta \in(0,1) .
$$

This property implies that every submatrix $A_{S}$ formed by combining at most $k$-columns in $A$ has its nonzero singular values bounded above by $1+\delta$ and below by $1-\delta$. In particular, (12) implies that a matrix $A$ cannot satisfy $R I P_{2, \delta, k}$ if $k \geq \operatorname{spark}(A)$.

Candès has shown, see [8, Thm. 1.1], that if $A \in R I P_{2, \delta, 2 k}$ with $\delta<\sqrt{2}-1$ all $x \in \mathbb{R}_{k}^{n}$ solve both (2) and (3). Moreover, there exists sensing matrices $A \in \mathbb{R}^{m \times n}$ which satisfy e.g. the $R I P_{2,1 / 4, k}$, where $k$ can be as large as $O(m / \log (m / n))$. This class includes matrices with i.i.d standard Gaussian or \pm 1 entries, random submatrices of the Fourier transform or other orthogonal matrices.

It has been shown recently [12] that binary matrices cannot satisfy $R I P_{2, k, \delta}$, unless the numbers of rows is $\Omega\left(k^{2}\right)$. Note that the best known explicit construction of (binary) compressed sensing matrices due to DeVore [14] yields $\Omega\left(k^{2}\right)$ measurements, which is worse than the bound $m=O(k \log (n / k))$.

Theorem 4.7. [12, Thm. 1] Let $A \in \mathbb{R}^{m \times n}$ be any $0 / 1$-matrix that satisfies $R I P_{2, k, \delta}$. Then

$$
m \geq \min \left\{\left(\frac{1-\delta}{1+\delta}\right)^{2} k^{2}, \frac{1-\delta}{1+\delta} n\right\} .
$$


For our particular $A$ defined in (4) with $\operatorname{spark}(A)=8$ we therefore obtain taking $m=3 d^{2}$ into account

Corollary 4.8. Let $\delta \in(0,1)$. Then a necessary condition for $A$ to satisfy the $R I P_{2, k, \delta}$ for all $k$-sparse vectors is that

$$
k \leq \min \left\{\frac{\sqrt{3}}{2} \cdot \frac{1+\delta}{1-\delta} d, 7\right\} .
$$

However, we cannot be sure that $A$ possesses the $R I P_{2,7, \sqrt{2}-1}$, unless we compute all singular values of all $A_{S},|S| \leq 7$ matrices. In case of a positive result we obtain $\ell_{0} / \ell_{1}$ equivalence for all less then 3 -sparse particle distributions, even in case of observation errors, since $R I P$ also implies stable recovery, provided that the observation error is small enough, compare [8, Thm. 1.2] for the "noisy" version result.

\subsection{Binary Matrices with $R I P_{1, k, \delta}$}

In [4] it is shown that a particular class of binary measurement matrices $A \in\{0,1\}^{m \times m}$, namely adjacency matrices of expander graphs, see the following Def. 4.1, satisfy a different form of restricted isometry property, the so-called $R I P_{1, k, \delta}$

$$
\forall x \in \mathbb{R}_{k}^{n}, \quad(1-\delta)\|x\|_{1} \leq\|A x\|_{1}=(1+\delta)\|x\|_{1}, \quad \delta \in(0,1) .
$$

Definition 4.1. A $(k, \epsilon)$-unbalanced expander is a bipartite simple graph $G=(A, B, E)$ with left degree $\ell$ such that for any $X \subset A$ with $|X| \leq k$, the set of neighbors $\mathcal{N}(X) \subset B$ of $X$ has size $|\mathcal{N}(X)| \geq(1-\epsilon) \ell|X|$.

The existence of expander graphs can be shown using the probabilistic method without explicitly constructing them, see $[4,24]$ and the references therein.

Conversely, any binary matrix with $\ell$ ones in each column and satisfying $R I P_{1, k, \delta}$ must be the adjacency matrix of an unbalanced expander graph, compare [4, Thm. 2].

The significance of $R I P_{1, k, \delta}$ is the following performance guarantee when reconstructing an arbitrary (not necessarily $k$-sparse) vector $x$ by solving (3).

Theorem 4.9. [4, Thm. 3] Let $A \in\{0,1\}^{m \times n}$ be the adjacency matrix of an unbalanced $(2 k, \epsilon)$-expander. Let $\alpha(\epsilon)=(2 \epsilon) /(1-2 \epsilon)$. Consider any two vectors $x, x^{*}$ such that $A x=$ $A x^{*}$ and $\|x\|_{1} \leq\left\|x^{*}\right\|_{1}$. Then

$$
\left\|x^{*}-x\right\|_{1} \leq \frac{2}{(1-2 \alpha(\epsilon))}\left\|x^{*}-x^{k}\right\|_{1}
$$

where $x^{k} \in \mathbb{R}_{k}^{n}$.

Proposition 4.10. Let be $A$ the adjacency matrix of an arbitrary bipartite graph with regular left degree $\ell$ and denote $\sigma=\operatorname{spark}(A)$. Then $A$ is the adjacency matrix of an $\left(\sigma-1,1-\frac{1}{\ell}\right)-$ unbalanced expander.

Proof. Let $X \subset\{1, \ldots, n\}$ and $|X|=k \leq \sigma-1$. Then $A_{X}$ is a overdetermined full rank matrix. In particular, there exist $k$ linearly independent rows in $A_{X}$, each of them having at least one nonzero entry. Hence $|\mathcal{N}(X)| \geq|X|=\ell\left(1-\left(1-\frac{1}{\ell}\right)\right)|X|$. 
The above proposition implies that $A$ from (4) is the adjacency matrix of an $\left(7, \frac{2}{3}\right)$ unbalanced expander. As a consequence we obtain exact recovery for every 3-sparse particle distribution in view of Thm. 4.9 and Prop. 4.1.

\subsection{Neighborly Polytopes}

Donoho and Tanner $[15,19]$ explained the $\ell_{0} / \ell_{1}$-equivalence phenomenon from the view point of convex neighborly polytopes. In contrast to sufficient conditions for exact recovery, this theory provides necessary conditions additionally.

We first summarize some terminology from convex polytopes theory and refer to [23] and [19] for more details. A linear inequality $c^{\top} x \leq d$ is called valid for a polytope $P \in \mathbb{R}^{n}$ if it is satisfied for all $x \in P$. A subset $F \subseteq P$ is called a face of $P$ if $F=\emptyset$ or $F=P$ (the improper faces), or

$$
F=P \cap\left\{x \mid c^{\top} x=d\right\}
$$

for some valid inequality $c^{\top} x \leq d$. Faces of dimension $k$ are called $k$-faces. Vertices, the extreme points of $P$, are the 0 -faces. A polytope $P$ is said to be outwardly $k$-neighborly if every subset of $k$ vertices not including 0 span a $(k-1)$-face, see [19], thus a outwardly $k$ neighborly polytope behaves like a simplex, at least from the viewpoint of it's lowdimensional faces (not including 0), since every $p$-dimensional face (not including 0 ) is simplicial, for $0 \leq p<k$.

The main result in [19] connects outward neighborliness to the question of uniqueness of any $k$-sparse nonnegative vector. Such a $k$-sparse vector $x^{*}$ will "live" on a $k$-face of the convex hull of the standard simplex in $\mathbb{R}^{n}$ and the origin, denoted by $\Delta_{0}^{n-1}$. If $A x^{*}$ will "survive" on a $k$-face of $A \Delta_{0}^{n-1}=\operatorname{conv}\left\{A_{\bullet}, \ldots, A_{\bullet}, n, 0\right\}$ then it will be the unique positive solution satisfying $A x=A x^{*}$. If $A x^{*}$ falls "inside" the "transformed" polytope $A \Delta_{0}^{n-1}$ then $x^{*}$ cannot be recovered by (5). For a outwardly $k$-neighborly polytope $A T_{0}^{n-1}$ this will never happen.

We will extend this result by the following simple observation.

Theorem 4.11. Let $A \in \mathbb{R}^{m \times n}$ be an arbitrary matrix. Then the following statements are equivalent:

(a) Every $k$-sparse nonnegative vector $x^{*}$ is the unique positive solution of $A x=A x^{*}$.

(b) The the convex polytope defined as the convex hull of the columns in A and the zero vector, i.e. $\operatorname{conv}\left\{A_{\bullet}, 1, \ldots, A_{\bullet}, n, 0\right\}$ is outwardly k-neighborly.

(c) Every nonzero nullspace vector has at least $k+1$ negative entries.

Proof. The equivalence of (a) and (b) is the main result in [19, Thm. 1].

$(c) \Rightarrow(a)$ : Now, let $x^{*}$ be a $k$-sparse vector. Any other (different) positive solution of $A x=A x^{*}$ must be of the form $x^{*}+v$ such that $x^{*}+v \geq 0$ and $v \in \operatorname{ker}(A) \backslash\{0\}$. Hence $I^{-}\left(x^{*}+v\right)=\emptyset$. This contradicts $\left|I^{-}\left(x^{*}+v\right)\right| \geq 1$ as claimed by (c).

$(a) \Rightarrow(c)$ : Conversely, lets assume that there exist a nonzero nullspace vector $v$ with $\left|I^{-}(v)\right| \leq$ $k$. We now define two nonnegative vectors $x^{1}$ and $x^{2}$ in the following way

$$
x_{i}^{1}= \begin{cases}v_{i}, & \text { if } i \notin I^{-}(v) \\ 0, & \text { otherwise }\end{cases}
$$



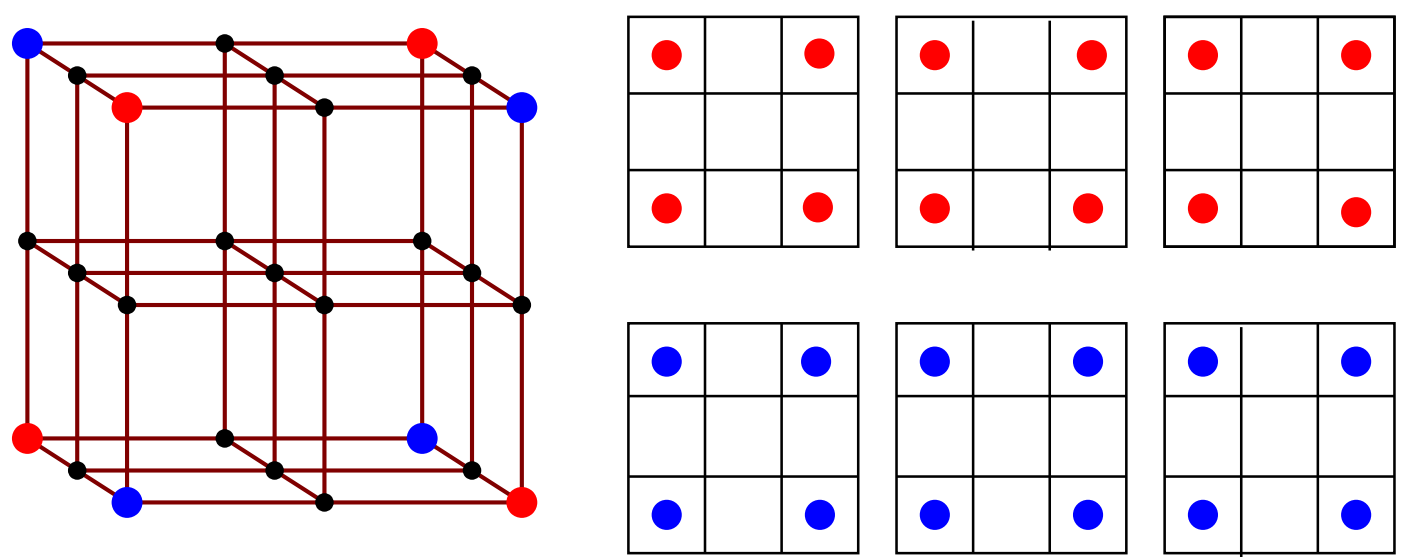

Figure 3: Two different non unique 4-sparse "particle" distributions in a $3 \times 3 \times 3$ volume, based on the construction in the proof of Thm. 4.11. Both configurations (represented by red and blue dots) yield the identical projections in all three directions.

and

$$
x_{i}^{2}= \begin{cases}-v_{i}, & \text { if } i \notin I^{-}(v) \\ 0, & \text { otherwise } .\end{cases}
$$

Since $x^{1}-x^{2}=v \neq 0$ we obtain two different solution to $A x^{1}=A x^{2}$ although $x^{2}$ is $k$-sparse. This completes the proof.

From Prop. 2.2 we know the existence of nullspace vectors with only 4 negative entries. This together with Thm 4.11 now yields

Corollary 4.12. The convex hull of the columns in matrix A defined in (4) and the zero vector, i.e. $\operatorname{conv}\left\{A_{\bullet}, 1, \ldots, A_{\bullet}, n, 0\right\}$ is outwardly 3-neighborly.

Hence, the maximal sparsity level $k$ such that $\ell_{0} / \ell_{1}$-equivalence holds for all $k$-sparse nonnegative vectors holds is 3 . Indeed, in a $d \times d \times d$ volume there are $\left(\begin{array}{l}d \\ 2\end{array}\right)^{3} 4$-sparse vector pairs with equal projections, compare Fig. (3).

\subsection{Nullspace Property}

Similar to the our simple observation in the previous section the authors in [13] derived sparse recovery conditions based on properties of the nullspace of $A$. In particular, they say that a matrix $A$ has the Null Space Property ${ }^{1}$ of order $k$ for $k>0$ for $\gamma>0$ if

$$
\left\|v_{S}\right\|_{1} \leq \gamma\left\|v_{S^{c}}\right\|_{1}
$$

for all sets $S$ of cardinality less then $k$ and $v \in \operatorname{ker}(A)$. In [13, Thm. 4.3] it is shown that if $A$ has the nullspace property of order $\geq k$ and $\gamma<1$ it is guaranteed that every $k$-sparse vector is the unique $\ell_{1}$-minimizer of (3). The nullspace property is a weaker version of the

\footnotetext{
${ }^{1}$ For convenience we slightly modified the original definition of the Null Space Property given in [13].
} 
restricted $\ell_{2}$-isometry property. Indeed, Cohen et al showed [13, Lem. 4.1] that if $A$ satisfies the $R I P_{2,3 k, \delta}$ then $A$ satisfies the nullspace property of order $2 k$ and $\gamma=\frac{\sqrt{2}}{2} \sqrt{\frac{1+\delta}{1-\delta}}$.

In independent work, Zhang [31] used the general concept of $k$-balanceness to study uniqueness of the $\ell_{1}$-minimizer. A subspace $X$ is $k$-balanced (in $\ell_{1}$-norm) if for any $S$ with $|S| \leq k$

$$
\left\|v_{S}\right\|_{1} \leq\left\|v_{S^{c}}\right\|_{1}
$$

holds for all $v \in X . X$ is called strictly $k$-balanced if the strict inequality holds. Hence, strict $k$-balanceness of the nullspace of $A$ implies the nullspace property of order $k$ with $\gamma<1$, thus, exact recovery. In fact, the author shows in [31] that $k$-balanceness of $\operatorname{ker}(A)$ is equivalent to $\operatorname{conv}\left\{ \pm A_{\bullet}, 1, \ldots, \pm A_{\bullet}, n, 0\right\}$ being (outwardly) $k$-neighborly. The latter is the analogous sufficient and necessary condition for recovery of all $k$-sparse vector when the vector might have different signs, compare [15].

In the nonnegative case Zhang showed [32] the equivalence of the (outwardly) $k$-neighborliness of the polytope $\operatorname{conv}\left\{A_{\bullet, 1}, \ldots, A_{\bullet, n}, 0\right\}$ and the notion of half $k$-balanceness of the nullspace of $A$. A subspace $X$ is half $k$-balanced (in $\ell_{1}$-norm) if for any $S$ with $|S| \leq k$

$$
\sum_{i \in S} v_{i} \leq\left\|v_{S^{c}}\right\|_{1}
$$

holds for all $v \in X . X$ is called strictly half $k$-balanced if the strict inequality holds. Hence, this different form of nullspace property for nonnegative vectors turns out to be sufficient and necessary condition for uniqueness of every $k$-sparse nonnegative vector, in view of the first part of Thm. 4.11. However, testing the nullspace property conditions on generic matrices is potentially harder than solving the combinatorial $\ell_{0}$ problem in (2) as it implies solving a combinatorial problem to compute $\gamma$. However, we can conclude that $A$ from (4) has the nullspace property of order 3 with $\gamma<1$, due to the previous observations. This ends the series of highly pessimistic conclusions concerning our particular $A$.

\section{Most Probably Unique Positive Solution}

\subsection{Weak Equivalence}

The concept of $\ell_{0} / \ell_{1}$ equivalence demands that for a given measurement matrix $A$, equivalence for all instances $(A, b)$ generated by any $k$-sparse vector holds. A weaker form of equivalence considers equivalence for most problem instances $(A, b)$. In [19] it is shown that a weaker form of neighborliness implies weak equivalence. The authors define a polytope $P$ to be $(k, \epsilon)$-weakly (outwardly) neighborly if, among all $k$-subsets of vertices (resp. among those not including 0), all except a fraction $\epsilon$ span $k-1$-faces of $P$.

The columns of $A$ are in general position if all subsets of $m$ columns of $A$ are linearly independent, thus $\operatorname{spark}(A)=m+1$. It is shown in [19] that if the columns of $A$ are in general position, weak neighborliness of $A \Delta_{0}^{n-1}=\operatorname{conv}\left\{A_{\bullet, 1}, \ldots, A_{\bullet, n}, 0\right\}$ is the same thing as saying that $A \Delta_{0}^{n-1}$ has at least $(1-\epsilon)$-times as many $(k-1)$-faces as $\Delta_{0}^{n-1}$. Thm. 2 in [19] shows the equivalence between $(k, \epsilon)$-weakly (outwardly) neighborliness and weak equivalence, i.e. uniqueness of all except a fraction $\epsilon$ of $k$-sparse nonnegative vectors. 
However, the columns of $A$ from (4) are not in general position. Besides, counting faces of polytopes is again a combinatorial problem.

To overcome this difficulty we appeal to the observation already made in Section 2. If the matrix obtained by reducing zero measurements and corresponding adjacent voxels is overdetermined and of full rank then the underlying solution which generated the sparse measurement vector must be unique. This is also a criterion of individual equivalence for a given problem instance $(A, b)$. Moreover, a critical sparsity level $k$ yielding weak equivalence for $A$ of most $k$-sparse nonnegative vectors can be derived by estimating the probability that $k$-columns are linearly independent with probability close to one, i.e $\operatorname{sig}_{k}(A) \approx 0$, and estimating the probability that the induced reduced matrix is overdetermined.

\subsection{Probability of $m_{\text {red }}(k) \geq n_{\text {red }}(k)$}

Sparse vectors $x$ give rise to sparse vectors $b=A x$. Based on the zero components of $b$ corresponding rows and columns can be removed from $A$, leading to a reduced matrix $A_{\text {red }} \in$ $\mathbb{R}^{m_{\text {red }}(k) \times n_{\text {red }}(k)}$. In this section, we estimate the expected dimension of the reduced matrix depending on the sparsity $k$ of $x$.

Lemma 5.1. Let $x \in\{0,1\}^{d^{3}}$ be a uniformly drawn $k$-sparse binary vector. Then the expected number of zero measurements in any of the there projection images approximately is

$$
\mathbb{E}[k, d]:=\frac{1}{d^{2 k}} \sum_{r=0}^{d^{2}} r\left(\begin{array}{c}
d^{2} \\
r
\end{array}\right)\left(d^{2}-r\right) ! S_{k, d^{2}-r},
$$

where $S_{n, k}$ denotes the Stirling number of the second kind.

Proof. Let $p: K \rightarrow R$ be any of the three projection directions considered as a function mapping $|K|=k$ particles onto $|R|=d^{2}$ pixels. We wish to determine the probability that $r$ pixels, corresponding to $r$ rows in the measurement vector $b$, remain "empty".

This probability is given by $\left|\Omega_{r}\right| /|\Omega|$, where $\Omega$ denotes the set of all projections $p$, i.e. $|\Omega|=$ $|R|^{|K|}$, and where $\Omega_{r} \subset \Omega$ contains functions $p$ mapping $k$ particles to $|R|-r$ pixels.

Assume $r$ "empty" pixels are fixed. Then only surjective mappings $p$ assign $k$ particles to all remaining $|R|-r$ pixels without leaving any additional pixel empty. The size of this set is $(|R|-r) ! S_{k,|R|-r}$ is [2]. Because there are $\left(\begin{array}{c}|R| \\ r\end{array}\right)$ ways to locate the $r$ zero pixels, we obtain

$$
\left|\Omega_{r}\right|=\left(\begin{array}{c}
|R| \\
r
\end{array}\right)(|R|-r) ! S_{k,|R|-r}
$$

Clearly, $|\Omega|=\sum_{r=0}^{|R|}\left|\Omega_{r}\right|$, and the expected number of zero pixels is $\mathbb{E}[k, d]=\sum_{r=0}^{|R|} r \frac{\left|\Omega_{r}\right|}{|\Omega|}$.

Remark 5.1. We point out that (15) is an approximation only, because we ignored the dependencies between particles due to the third dimension. Consequently, the numbers (17) determined below as a function of $\mathbb{E}[k, d]$ are approximations as well. 
Proposition 5.2. Let $x \in\{0,1\}^{d^{3}}$ be a uniformly drawn $k$-sparse binary vector. Then the expected values of the dimension of the reduced matrix $A_{\text {red }}$ approximately are:

$$
\begin{aligned}
& m_{\text {red }}(k) \approx m-3 \mathbb{E}[k, d] \\
& n_{\text {red }}(k) \approx d^{3}-3 \mathbb{E}[k, d] \cdot d+3 \frac{\mathbb{E}[k, d]^{2}}{d}-\left(\frac{\mathbb{E}[k, d]}{d^{2}}\right)^{3} d^{3} .
\end{aligned}
$$

Figure 4 illustrates that these estimates are reasonably tight.

Proof. The estimate (17a) is based on our assumption that $x$ is uniformly distributed. We simply subtract from the total number of pixels (rows) the expected number of zero measurements in all three projections due to Lemma 5.1, thus obtaining the expected number of zero components of the observed vector $b$.

Concerning (17b), any zero component of the vector $b$ marks voxels in the volume along the corresponding projection ray, and corresponding columns in $A$, to be removed from $A$. $n_{\text {red }}(k)$ is the number of voxels (columns) not removed by any projection. To estimate the expected value of this number, we have to take into account that projection rays intersect.

Based on the expected number $\mathbb{E}[k, d]$ of zero pixels in any of the three projections - see (15), we compute:

1. Each single projection removes $\mathbb{E}[k, d] \cdot d$ voxels.

2. Consider a pair of projections, e.g. the $x / z$-projection and the $y / z$-projection. Fix the common $z$-coordinate. There are $\mathbb{E}[k, d] / d$ zero pixels in each of the two corresponding rows of the two projection images, eliminating together $(\mathbb{E}[k, d] / d)^{2}$ voxels because all projection rays corresponding to the two sets of zeros mutually intersect. As there are $d$ possible values of $z$, it follows that each pair of projections removes $d(\mathbb{E}[k, d] / d)^{2}=$ $(\mathbb{E}[z])^{2} / d$ voxels.

3. The probability that any fixed voxel projects to a zero in any fixed projection is $\mathbb{E}[k, d] / d^{2}$, due to Lemma 5.1. Consequently, the expected number of voxels removed by all three projections is $\left(\frac{\mathbb{E}[k, d]}{d^{2}}\right)^{3} d^{3}$.

$n_{\text {red }}(k)$ corresponds to the number of voxels for which all three conditions above do not hold, which due to the inclusion-exclusion principle is given by (17b).

Comparing (17a) and (17b) shows that more columns are removed than rows, depending on the expected number $\mathbb{E}[k, d]$ of vanishing components of $b=A x$. Hence for a sufficiently $k$-sparse vector $x$ the reduced matrix $A_{\text {red }}$ leads to an overdetermined system with $m_{\text {red }}(k) \geq$ $n_{\text {red }}(k)$. Solving the polynomial $m_{\text {red }}(k)=n_{\text {red }}(k)$ according to (17) in the variable $\mathbb{E}[k, d]$ for the root in the admissible interval $\left[1, d^{2}\right]$, we find that this will hold on the average for $k$-sparse vectors $x$ that generate at least

$$
\mathbb{E}[k, d] \approx\left(1-\sqrt{\frac{3}{d}}\right) d^{2}
$$



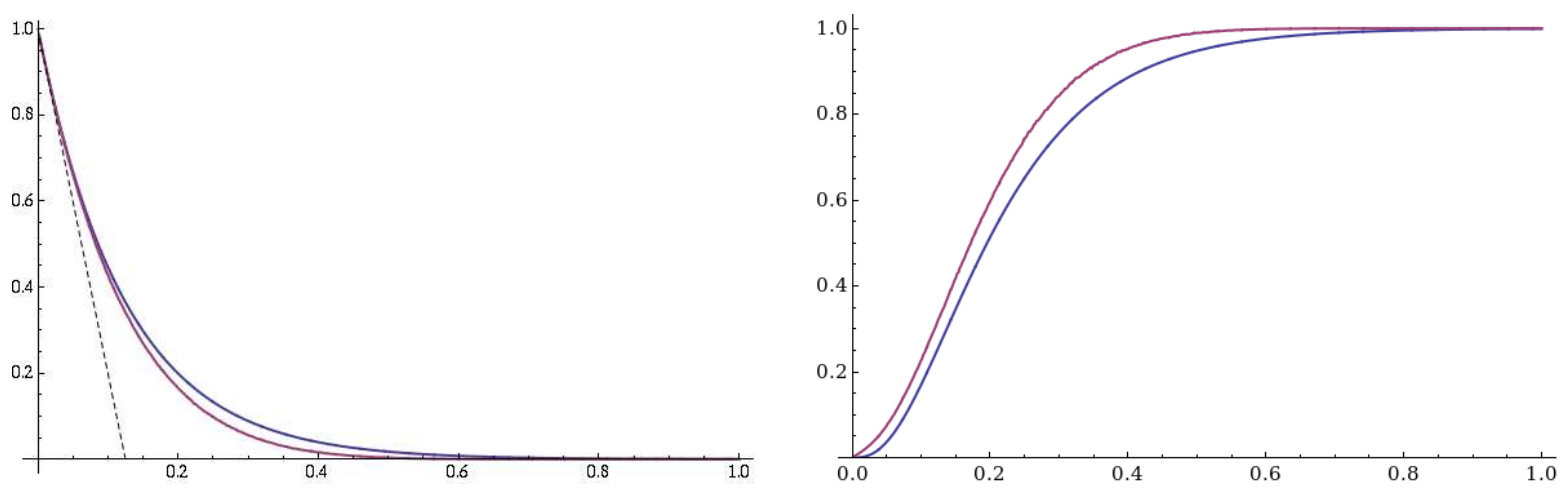

Figure 4: Left: Expected number of zero components of the measurement vector $b=A x$, generated by a $k$-sparse random vector $x$. The blue curve shows $3 \mathbb{E}[k, d] / m$ as a function of $\frac{k}{d^{3}}$ due to (15). These numbers are related to the expected number $m_{\text {red }}(k)$ of rows of the reduced matrix $A_{\text {red }}$ by (17a). The red curve shows the corresponding empirical means computed for $d=8$, i.e. for the matrix $A \in \mathbb{R}^{192 \times 512}$, and 1000 trials for each value of $k$. The dashed curve shows the asymptotic $1-\frac{k}{m}$ for small values of $k$. Right: Expected number of columns of the reduced matrix $A_{\text {red }}$. The blue curve shows $\frac{n_{\text {red }}(k)}{n}$ as a function of $\frac{k}{n}$, with $n_{\text {red }}(k)$ given by (17b). The red curve shows the corresponding empirical curve obtained by simulations as described above.

zero entries in each projection. Figure 5 shows the corresponding critical values of the sparsity parameter $k=k(d)$, numerically determined by solving $m_{\text {red }}(k)=n_{\text {red }}(k)$ resp. (18), as a function of the problem size $d$. The log-log plot in the right panel of Figure 5 indicates quite accurately the power law

$$
k(d) \approx 3.54 d^{1.34}
$$

\section{Towards an improvement - Perturbation of $A$}

\subsection{Increasing Spark}

Having the previous results in mind we further address the question of improving the properties of $A$ from (4) with respect to the overall objective: $\ell_{0} / \ell_{1}$-equivalence. The weak performance of $A$ rests upon the small spark of $A$. In order to increase the maximal number $s$ of columns such that all $s$ (or less) column combinations are linearly independent we add to the entries of $A$ a small perturbation.

We will keep in mind the following result which might be well known.

Lemma 6.1. Let $B \in \mathbb{R}^{m \times n}$ be any matrix of rank $r, \sigma_{1} \geq \sigma_{2} \geq \cdots \geq \sigma_{r}>0$ its singular values and $B=U \Sigma V^{\top}$ is singular value decomposition, where

$$
\Sigma=\left(\begin{array}{cc}
\Sigma_{r} & 0 \\
0 & 0
\end{array}\right)
$$



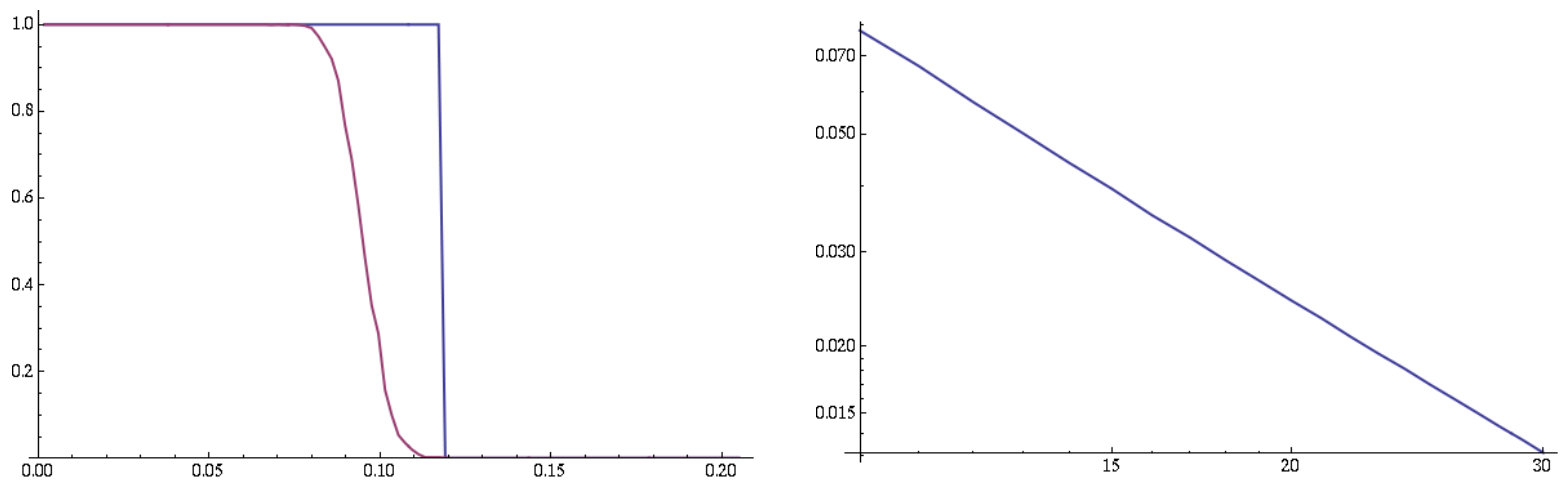

Figure 5: Left: Critical value of $k / d^{3}$, for $d=8$, below of which the reduced matrix $A_{\text {red }}$ satisfies $m_{\text {red }}(k) \geq n_{\text {red }}(k)$ with high probability. The blue curve shows the estimate based on Prop. 5.2. The red curve shows the empirical probability based on simulations as described in the caption of Fig. 4. Right: Critical value of $k / d^{3}=k(d) / d^{3}$ as a function of the problem size $d$, according to (17). The log-log plot indicates the power law (19).

with $\Sigma_{r}=\operatorname{diag}\left(\sigma_{1}, \ldots, \sigma_{r}\right)$. If $\|E\|<\sigma_{r}$ then $\operatorname{rank}(B+E) \geq \operatorname{rank}(B)$. Moreover, if we denote by

$$
U^{\top} E V=:\left(\begin{array}{ll}
E_{11} & E_{12} \\
E_{21} & E_{22}
\end{array}\right)
$$

then

$$
\operatorname{rank}(B+E)=\operatorname{rank}(A)+\operatorname{rank}(S)
$$

where $S$ is the Schur complement $E_{22}-E_{21}\left(\Sigma_{r}+E_{11}\right)^{-1} E_{12}$ of

$$
\left(\begin{array}{cc}
\Sigma_{r}+E_{11} & E_{12} \\
E_{21} & E_{22}
\end{array}\right)
$$

Proof. In view of our assumption we also have $\left\|E_{11}\right\|<\sigma_{r}$ since $U, V^{\top}$ are orthogonal. Hence $\left\|\Sigma_{r}^{-1} E_{11}\right\|<1$ holds, which also implies the nonsingularity of $\Sigma_{r}+E_{11}$. By writing

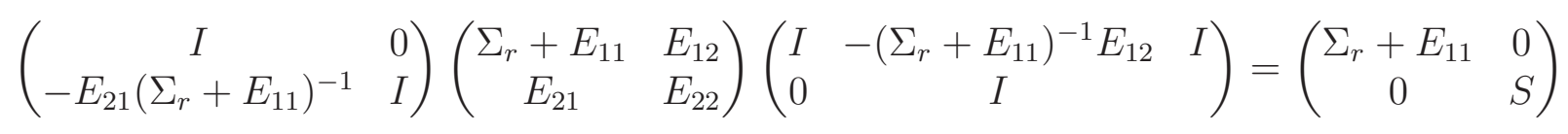

we obtain he desired result (20).

We stress that the above result holds for every matrix $E$. However, we are interested in matrices $E$ having the same sparsity structure like $A$.

We conjecture that the rank of every perturbed submatrix $A$ will grow by a factor $O(1)$. By perturbing $A$ we will "eliminate" all 8-column combinations (the column sets corresponding to nonzero entries in the nullspace basis vectors in $N$, compare Prop. 2.2). By elimination we meen that the perturbed 8-tuples will have complete rank since the unperturbed clearly have rank 7 since $\operatorname{spark}(A)=8$. Moreover, all $k$-linearly independent column sets of $A$ can be obtained by combining linearly independent 8-tuples. By a similar argument most such $k$-dependent columns in $A$ can be turned out in independent ones by simply perturbing their entries. This suggests that $\operatorname{spark}(\tilde{A})$ will grow proportional to the rank of $A$. The numerical results in Section 7 suggest the power law $\operatorname{spark}(\tilde{A}(d))=O\left(d^{2}\right)$, compare Fig. 7. 


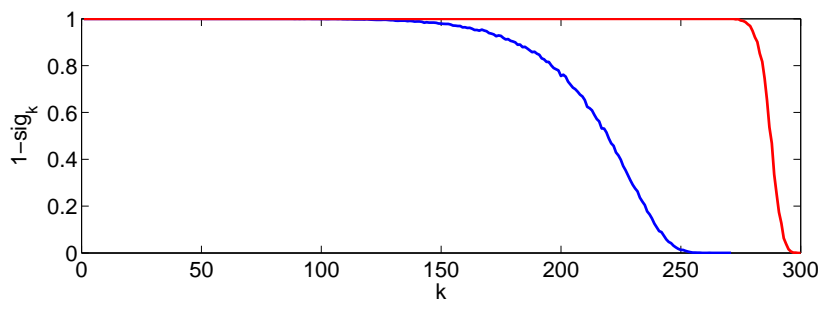

Figure 6: $1-\operatorname{sig}_{k}(A)$ versus perturbed $1-\operatorname{sig}_{k}(\tilde{A})$ for $d=10$; Empirical probability obtained from 100000 trials that $k$ columns are linearly independent.

Remark 6.1. For $A \in \mathbb{R}^{m \times n}$, let $\left\{\sigma_{1}, \sigma_{2}, \ldots, \sigma_{m}\right\}$ and $\left\{\tilde{\sigma}_{1}, \tilde{\sigma}_{2}, \ldots, \tilde{\sigma}_{m}\right\}$ be all singular values (nonzero as well as any zero ones) for $A$ and $\tilde{A}=A+E$, respectively. Then

$$
\left|\sigma_{i}-\tilde{\sigma}_{i}\right| \leq\|E\|_{2} \quad \text { for each } \quad i=1,2, \ldots, m .
$$

By choosing $E$ properly it seems possible to "adjust" the singular values of $A$ such that $\tilde{A}$ will satisfy the $R I P_{2}$ property. We intend to investigate this further in order to obtain recovery results that are stable in the presence of errors in TomoPIV measurements.

\subsection{How Neighborly will be the Perturbed Matrix?}

In Section 4 we presented several concepts which quantify the recovery performance of a given matrix $A$. Among these $k$-neighborliness and the nullspace property of order $k$ are necessary and sufficient conditions which guarantee uniqueness of every $k$-sparse positive vectors. In order to address the question of equivalence between (2) and (5) for $\tilde{A}$ we consider neighborliness of $\tilde{A} \Delta_{0}^{n-1}$.

Assume that by perturbing the nonnegative entries of $A$ we obtained an substantially increased spark $\tilde{\sigma}:=\operatorname{spark}(A+E)$. Set $\tilde{A}:=A+E$ and note that $\tilde{a}_{i j}=0$ iff $a_{i j}=0$.

Theorem 6.2. The convex hull of the columns in the matrix perturbed matrix $\tilde{A}$ and the zero vector, i.e. $\operatorname{conv}\left\{\tilde{A}_{\bullet}, 1, \ldots, \tilde{A}_{\bullet, n}, 0\right\}$ is at least outwardly $\left(\frac{\tilde{\sigma}}{3}-1\right)$-neighborly.

Proof. We will show that every nonzero nullspace vector has at least $\tilde{\sigma} / 3$ negative entries. Then Thm. 4.11 will provide the desired result. Let $v \in \operatorname{ker}(\tilde{A}) \backslash\{0\}$ and denote by $S=$ $\operatorname{supp}(v)$. Clearly,

$$
|S| \geq \tilde{\sigma}
$$

and

$$
|\mathcal{N}(S)| \geq \tilde{\sigma}
$$

where $\mathcal{N}(S)=\left\{i \in\{1, \ldots, m\} \mid \tilde{a}_{i j}>0, j \in S\right\}$ indexes all neighbors of $S$. In view of $S=I^{-1}(v) \cup I^{+}(v)$ and $v \in \operatorname{ker}(\tilde{A})$, we have

$$
\mathcal{N}\left(I^{-}(v)\right)=\mathcal{N}\left(I^{+}(v)\right)=\mathcal{N}(S)
$$

since it is not possible to find a voxel corresponding to a negative entry in $v$ indexed by $I^{-}(v)$, or a voxel corresponding to a positive entry in $v$ indexed by $I^{+}(v)$, that is not connected to 
both sets of rows $\mathcal{N}\left(I^{-}(v)\right)$ and $\mathcal{N}\left(I^{+}(v)\right)$, since otherwise $\tilde{A} v \neq 0$ in view of $\tilde{a}_{i j} \geq 0$. Summarizing we obtain

$$
\left|\mathcal{N}\left(I^{-}(v)\right)\right|=|\mathcal{N}(S)| \geq \tilde{\sigma} .
$$

On the other hand, since each voxel is connected to exactly 3 rows we have

$$
\left|\mathcal{N}\left(I^{-}(v)\right)\right| \leq 3\left|I^{-}(v)\right|
$$

Combining (24) and (25) we obtain the desired result.

This guarantees exact recovery by (5) via $\tilde{A}$ for at least all $(\tilde{\sigma} / 3-1)$-sparse nonnegative vectors.

We stress that is is possible to obtain a good upper bounds on the spark of an arbitrary matrix $A$ by computing first its row echelon (which can be done efficiently if $A$ is sparse) and then obtain a sparse nullspace vector from its row echelon.

\subsection{Unique Solution of the Reduced System}

Equivalence for most problem instances can be obtained by similar arguments as in Section 5.2. The critical value of $k$ such that an $k$-sparse vector with uniform distributed nonzero entries induces a overdetermined reduced system is again $k(d) \approx 3.54 d^{1.34}$. Then a lower bound to the critical value $k$ such that an $k$-sparse nonnegative vector with uniformly distributed nonzero entries is most probably unique if

$$
k(d) \geq \min \left\{3.54 d^{1.34}, 2.7 d^{2}\right\}
$$

where we assumed that $2.7 d^{2}$ or less columns combinations are most probably unique based on the results in Fig. 7.

\section{Numerical Experiments}

\subsection{Phase Transitions}

In this section we inspect empirical bounds on the required sparsity that guarantee exact reconstruction and critical parameter values that yield a performance similar to the settings considered in compressed sensing (e.g. $[15,19,16])$.

These parameter values allow us to answer the question how sparse a vector should be (particle density) such that $\ell_{0}$-minimization can be solved by $\ell_{1}$-minimization or simply by the linear program (5).

In analogy to [16] we assess the so called phase transition $\rho$ as a function of $d$, which is reciprocally proportional to the undersampling ratio $\frac{m}{n} \in(0,1)$. We consider $d \in\{3, \ldots, 55\}$, the corresponding matrix $A \in \mathbb{R}^{3 d^{2} \times d^{3}}$ from (4) and it's perturbed version $\tilde{A}$ and the sparsity as a fraction of $m=3 d^{2}, k=\rho m$, for $\rho \in(0,1)$.

This phase transition $\rho(d)$ indicates the necessary ratio $\frac{m}{n}$ to recover a $k$-sparse solution with overwhelming probability. More precisely, if $\|x\|_{0} \leq \rho(d) \cdot m$, then with overwhelming probability the $\ell_{0}$-problem of finding the $k$-sparsest solution can be solved by the LP (5). 
For Gaussian matrices there are precise values of $\rho(d)$, see $[16,19]$, which can be computed analytically.

Relevant for TomoPIV is the setting $d \approx 1024$. In the case of severe undersampling, i.e. as $d \rightarrow \infty$, a strong asymptotic threshold $\rho_{S}(d) \approx\left(2 e \log (2 \sqrt{\pi} d / 3)^{-1}\right.$ and weak asymptotic threshold

$$
\rho_{W}(d) \approx \frac{1}{2 \log \left(\frac{d}{3}\right)}
$$

holds for Gaussian matrices $A_{G}$ and nonnegative signals, where we have taken into account $A_{G} \in \mathbb{R}^{3 d^{2} \times d^{3}}$. The weak threshold says that $\rho_{W}(d) \cdot m$-sparse nonnegative vectors are typically the unique solutions of $A x=b$ while for the strong one equivalence between (2) and (5) holds for all $\rho_{S}(d) \cdot m$-sparse signals.

In view of Section 4, the strong threshold for $A$ from (4) equals 3 for all $d$, while for the perturbed matrix we can lower bound the strong threshold according to Thm. 6.2 by

$$
\rho_{S}(d) \geq \frac{\operatorname{spark}(\tilde{A}(d))}{3}-1
$$

Since $\operatorname{spark}(\tilde{A})$ will grow with $d$, we obtain an improvement over the constant strong threshold for the unperturbed matrix $A$. Verifying the strong threshold for $\tilde{A}$ empirically would be NP-hard. However, it is possible to verify the weak thresholds empirically by running tests on a random set of examples.

\subsection{Numerical Results}

For each $d \in\{3, \ldots, 55\}$ we generated $A$ according to (4) and $\tilde{A}$ by slightly perturbing it's entries. $\tilde{A}$ has the same sparsity structure as $A$, but random entries drawn from the standard uniform distribution on the open interval $(1,1.001)$. We have tried different perturbation levels, all leading to similar results. Thus we adopted this interval for all presented results.

Then for $\rho \in[0,1]$ a $\rho m$-sparse binary vector was generated to compute the right hand side measurement vector and for each $(d, \rho)$-point 100 random problem instances were generated.

The empirical probability that $k=\rho m$ columns of $A$ or $\tilde{A}$ are linearly independent for each parameter combination is presented in Fig. 7, while the probability that a $k=\rho m$-sparse vector can be recovered by the LP (5) is illustrated in Fig. 8, Fig. 9 and Fig. 10. Two slices of a phase transition plot for $d=50$ and $d=100$ are presented in Fig. 11. A threshold-effect is clearly visible in all figures exhibiting parameter regions where the probability of exact reconstruction is close to one. We refer to the figure captions for detailed explanations.

\section{Conclusion and Further Work}

The reconstruction of particle volume function from few projections can be modeled as finding the sparsest solution of an underdetermined linear system of equations, since the original particle distribution can be well approximated with only a very small number of active basis functions relative to the number of possible particle positions in a $3 \mathrm{D}$ domain. In general the search for the sparsest solution is intractable (NP-hard), however. The newly developed 
theory of Compressed Sensing showed that one can compute via $\ell_{1}$-minimization or linear programming the sparsest solution for underdetermined systems of equations provided that the coefficient matrix (also called measurement ensemble) satisfies certain conditions. Testing these conditions on generic matrices is often harder than solving the combinatorial $\ell_{0}$-problem in (2) as it also implies solving a combinatorial problem which is intractable given the huge dimensionality of the measurement matrix within the TomoPIV setting. However, we showed in the present work that all currently available recovery conditions predict an extremely poor performance of the TomoPIV measurement ensemble when we restrict to a simple but realistic setup geometry. On average, such matrices perform approximately five to ten times worse then Gaussian matrices which allow for maximal sparsity such that for all less sparse vectors exact recovery is still guaranteed. However, when we slightly perturb the entries of such an degenerate measurement matrix we can boost both worst case and expected reconstruction performance. Then the particle density can be increased by a factor of three while preserving the number of measurements. The theoretical analysis within this work suggests that a similar procedure can applied to an arbitrary sparse matrix with bad reconstruction performance. We will investigate this issue further for adjacency matrices of expander graphs with bad expansion property.

\section{Acknowledgement}

The authors gratefully acknowledge financial support from the German Science Foundation (DFG), grant SCHN/457-10. 


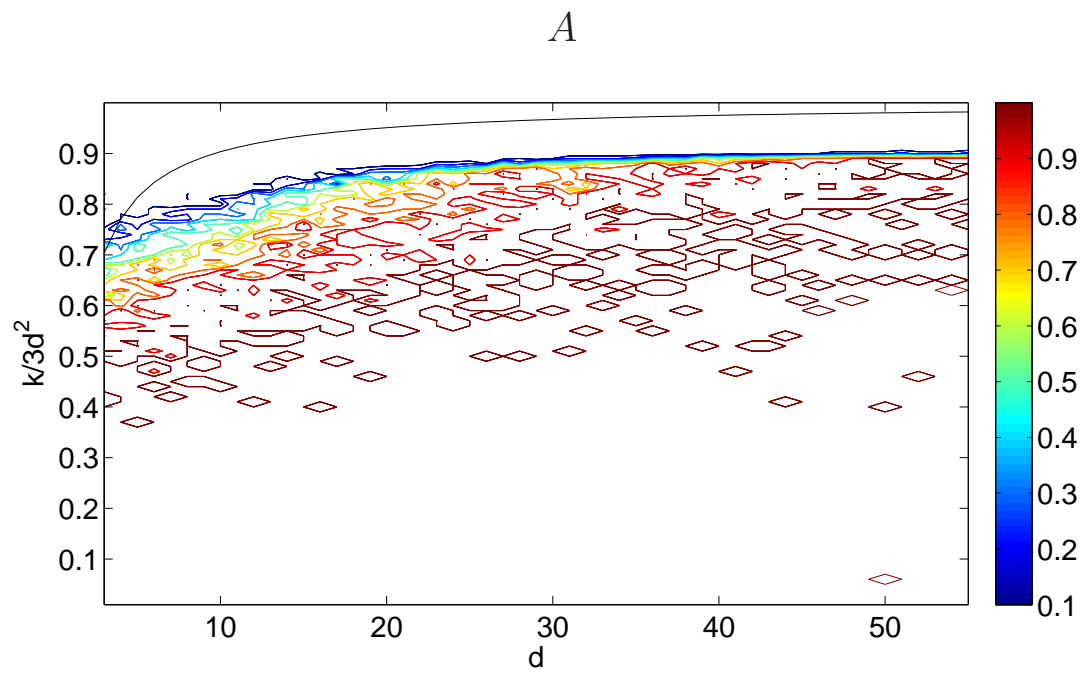

$\tilde{A}$

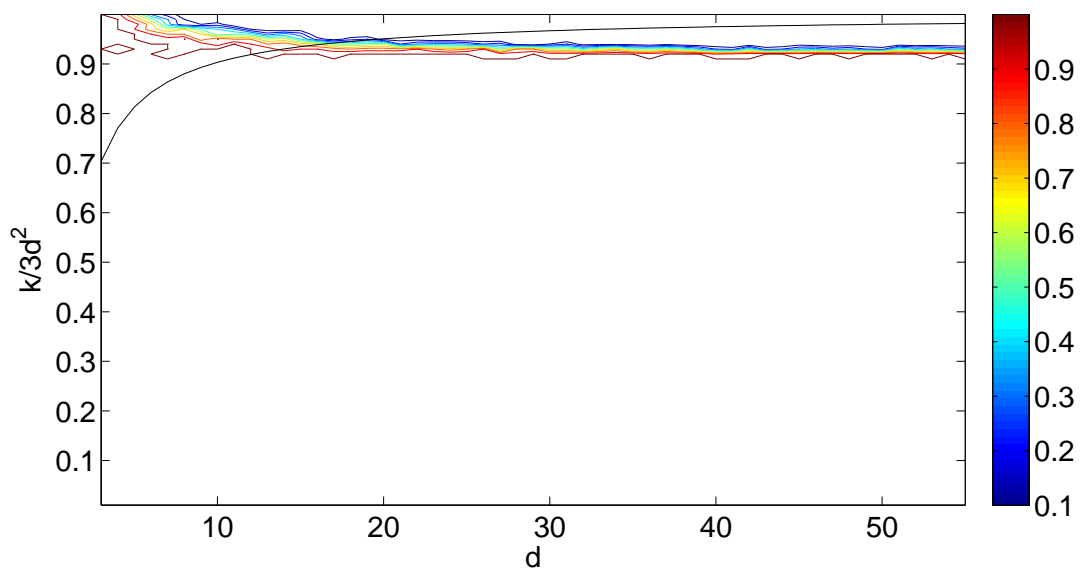

Figure 7: Top: Probability that $\rho m$ column combinations of $A$ are linearly independent. Bottom: Probability that $\rho m$ column combinations of the perturbed matrix $\tilde{A}$ are linearly independent. The black curve depicts the scaled rank of matrix $A$ as a function of $d$. The lower plot suggests that most $3 \cdot 0.9 d^{2}=2.7 d^{2}$ column combinations of the perturbed matrix $\tilde{A}$ are linearly independent with probability one. On the other hand, this can be claimed only for three times less columns of $A$. 

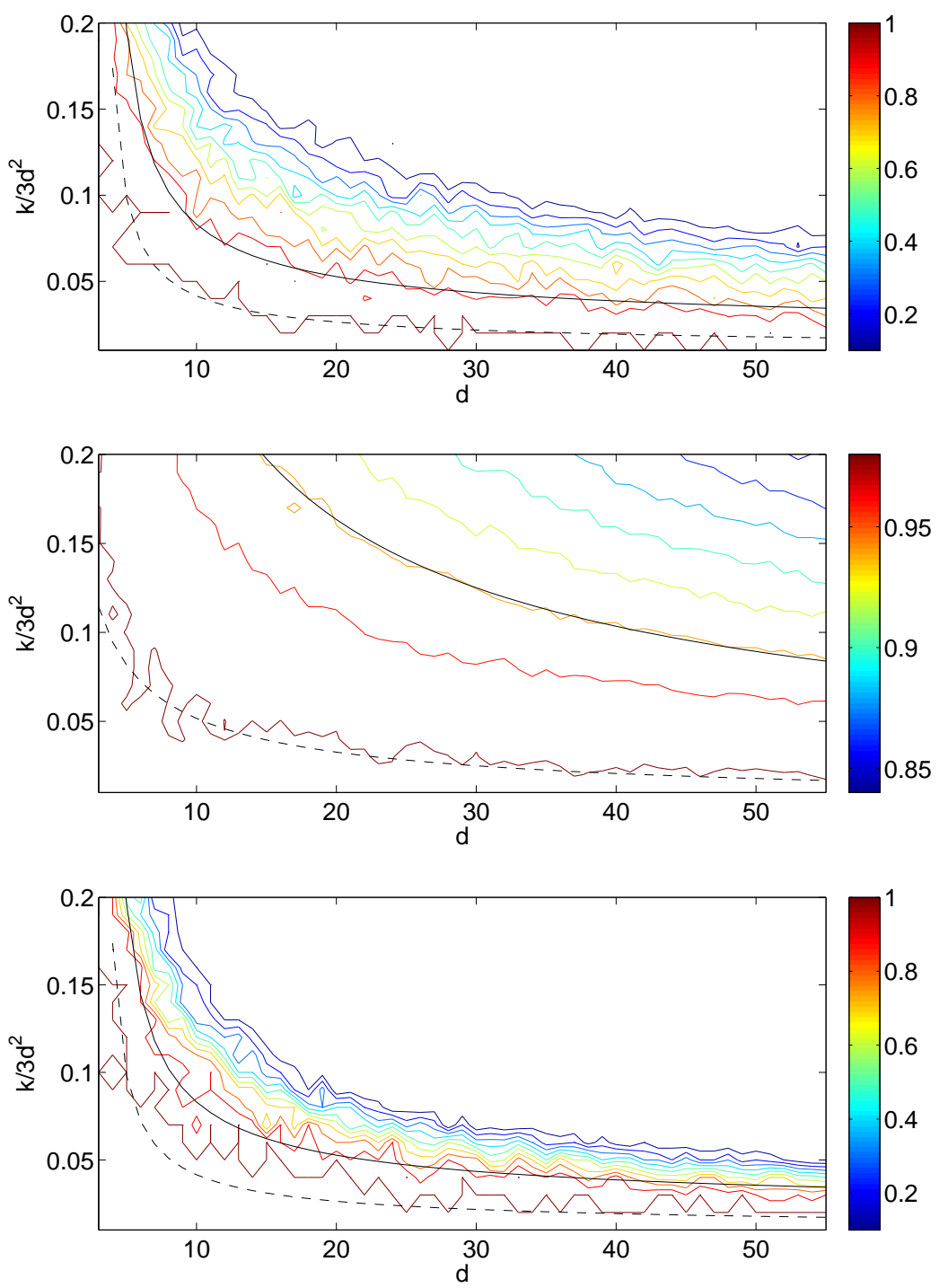

Figure 8: Results for matrix $A$ from (4). Top: Probability of correct recovery by linear programming of a random particle distribution that can be expressed with exactly $k=\rho m$ basis functions as a function of $d$. The solid black curve depicts $0.2 \rho_{W}(d)$ and the dashed one $0.1 \rho_{W}(d)$, where $\rho_{W}$ is the weak phase transition (26) of linear programming, but for Gaussian random matrices. The results indicate that $A$ from (4) performes ten times worse in recovering most sparse nonnegative signals. Middle: Probability that the reduced matrix $A$ obtained by eliminating zero measurements and corresponding adjacent voxels is overdetermined along with the estimated critical sparsity level $3.54 d^{1.34}$ relative to the number of measurements as a function of $d$ (solid black line), see (19). Ten times the dashed line equals the solid one. Bottom: Probability that a random $k=\rho m$ particle distribution induces an overdetermined and full rank reduced matrix. The results not only indicate that the reason for successful recovery in case of $A$ are full rank overdetermined reduced matrices, but also that solving just an overdetermined linear system might be more stable than linear programming, when the solution is known to be nonnegative. Here, the solid and dashed black curve depict again $0.1 \rho_{W}(d)$ and $0.2 \rho_{W}(d)$. 

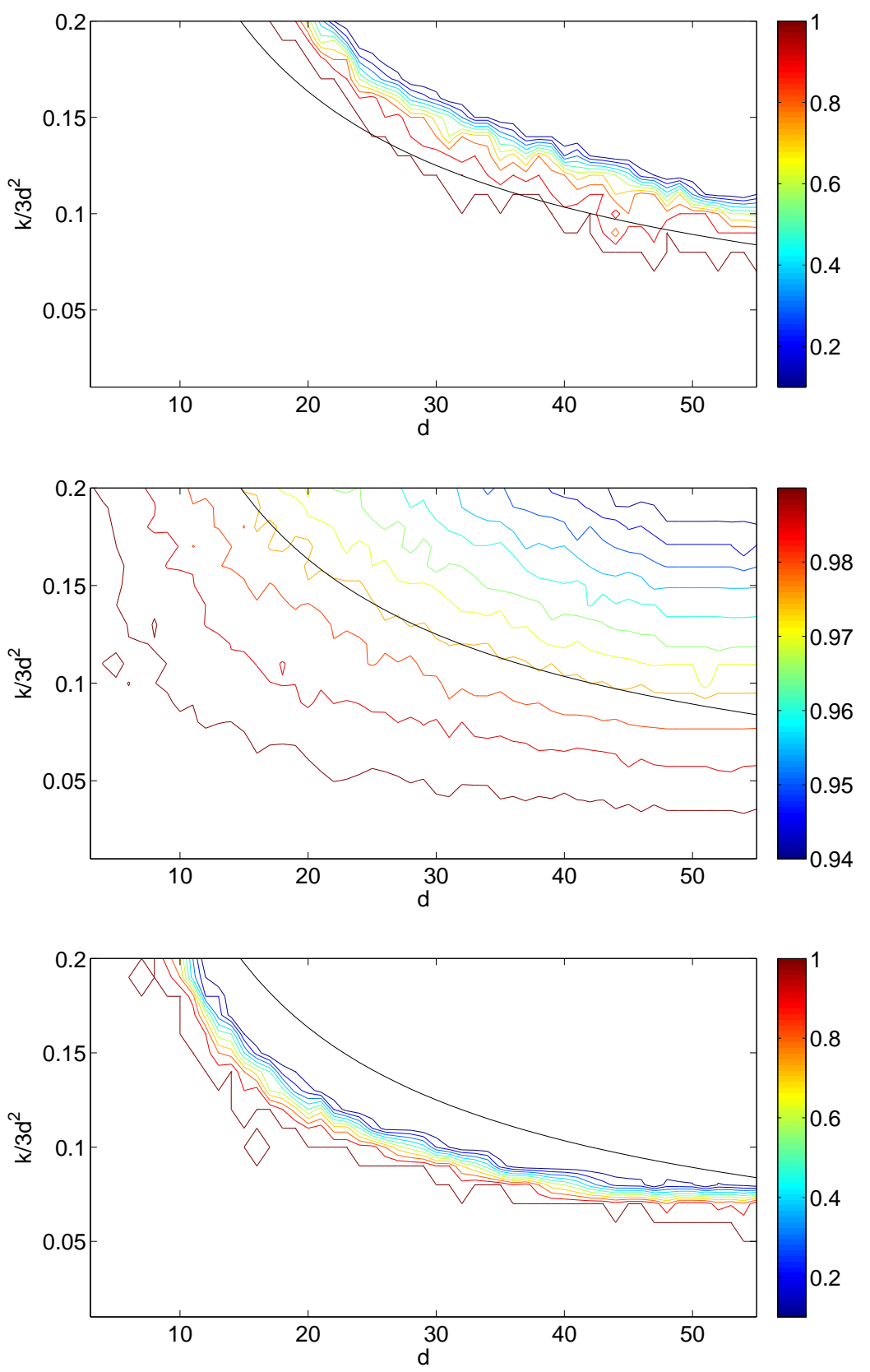

Figure 9: Results for the perturbed matrix $\tilde{A}$. Top: Probability of correct recovery of a $k=$ $\rho m$ sparse binary vector as a function of $d$. The solid black curve depicts $1.18 d^{-0.66}$, compare (19) and middle figure. Middle: Probability that the reduced matrix $A_{\text {red }}$ is overdetermined along with the estimated relative critical sparsity level $1.18 d^{-0.66}$ (solid black line) which induces overdetermined reduced matrices $\tilde{A}_{\text {red }}$. Bottom: Probability that a random $k=\rho m$ particle distribution induces an overdetermined and full rank reduced matrix along with the black curve $1.18 d^{-0.66}$. In case of the perturbed matrix $\tilde{A}$ exact recovery is possible beyond overdetermined reduced matrices. 

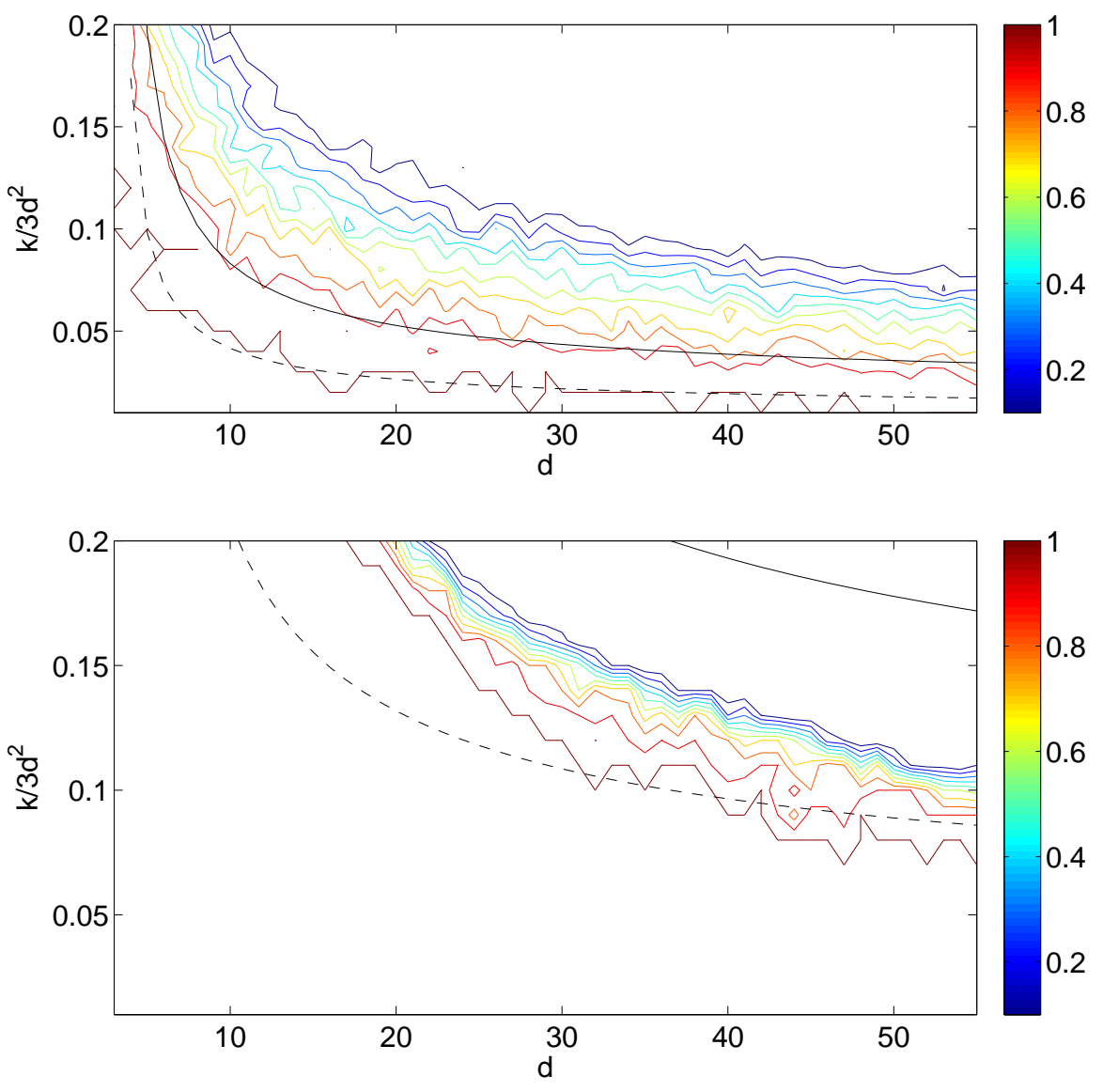

Figure 10: Recovery via $A$ from (4) (top) versus recovery via the perturbed matrix $\tilde{A}$ (bottom). Top: Success and failure empirical phase transition for $A$ along with $0.1 \rho_{W}(d)$ (dashed) and $0.2 \rho_{W}(d)$ (solid). Bottom: Success and failure empirical phase transition for the perturbed matrix $\tilde{A}$ along with $0.5 \rho_{W}(d)$ (dashed) and $\rho_{W}(d)$ (solid), compare (26). The results indicate that $\tilde{A}$ performs three times better in recovering $\rho m$ sparse vectors by the LP (5). 
(a) $d=50, A$

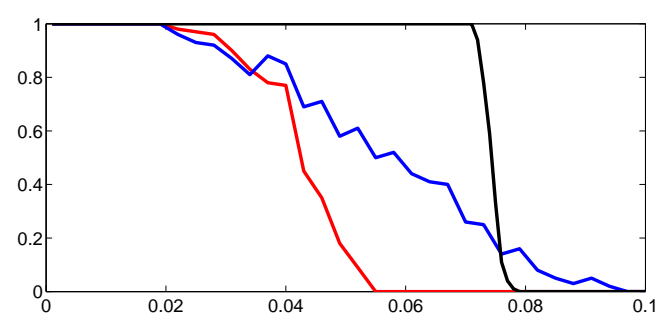

(c) $d=100, A$

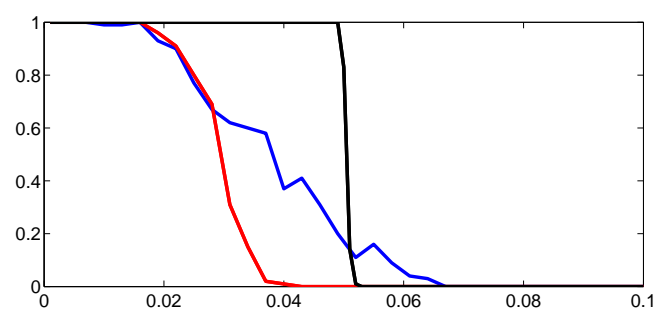

(b) $d=50, \tilde{A}$

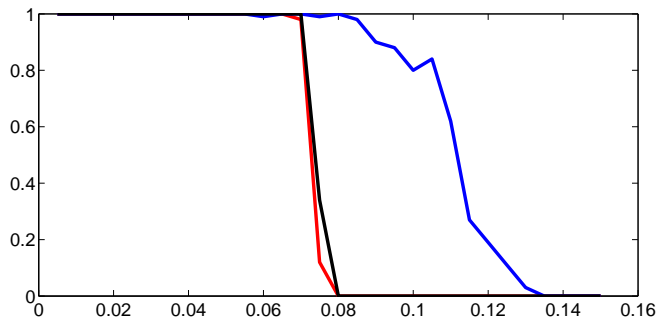

(d) $d=100, \tilde{A}$

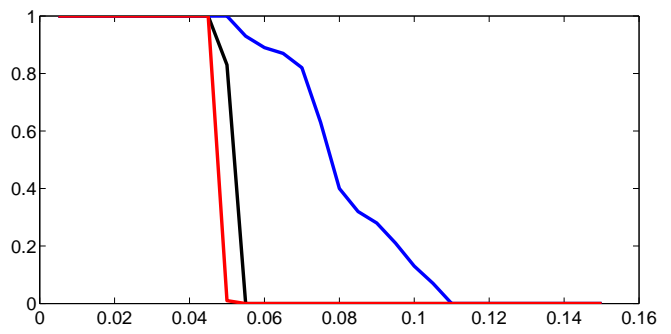

Figure 11: Slices through the contourplots Fig. 8 (top) and Fig. 9 (top); $A$ versus the perturbed matrix $\tilde{A}$ for $d=50$ and $d=100$. The blue line depicts the probability (as function of $\rho$ ) that a $\rho m$-sparse binary vector is recovered exactly by the LP (5). The red line illustrates the probability that a $\rho m$-sparse binary vector induces an overdetermined reduced matrix of full rank while the black line plots the probability that the reduced matrix is just overdetermined and not necessary of full rank. Here again $\tilde{A}$ performs three times better. 


\section{Appendix}

\section{Proof of Proposition 2.2}

Proof. (a) Note first that $\mathbf{1}_{d}^{\top}\left(\begin{array}{c}-\mathbf{1}_{d-1}^{\top} \\ I_{d-1}\end{array}\right)=0$ and compute

$$
\begin{aligned}
& A N=\left(\begin{array}{c}
\mathbf{1}_{d}^{\top} \otimes I_{d} \otimes I_{d} \\
I_{d} \otimes \mathbf{1}_{d}^{\top} \otimes I_{d} \\
I_{d} \otimes I_{d} \otimes \mathbf{1}_{d}^{\top}
\end{array}\right)\left(\left(\begin{array}{c}
-\mathbf{1}_{d-1}^{\top} \\
I_{d-1}
\end{array}\right) \otimes\left(\begin{array}{c}
-\mathbf{1}_{d-1}^{\top} \\
I_{d-1}
\end{array}\right) \otimes\left(\begin{array}{c}
-\mathbf{1}_{d-1}^{\top} \\
I_{d-1}
\end{array}\right)\right)
\end{aligned}
$$

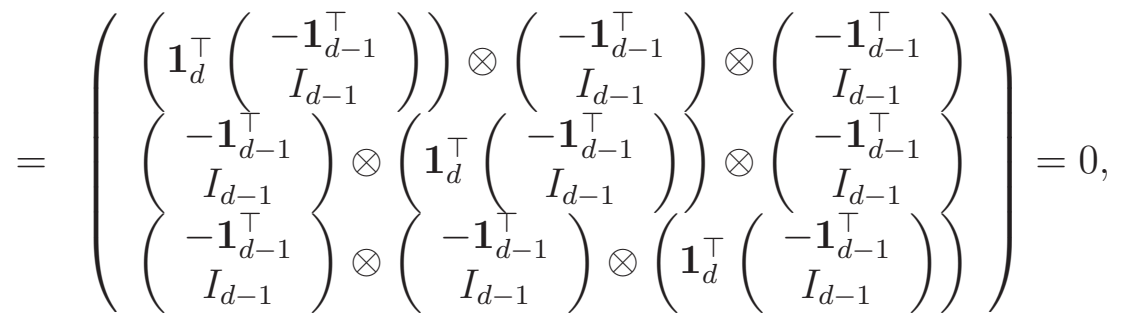

using the mixed product rule $\left(B_{1} \otimes C_{1}\right)\left(B_{2} \otimes C_{2}\right)=\left(B_{1} B_{2}\right) \otimes\left(C_{1} C_{2}\right)$, compare [22].

(b) Every column in $N$ from (9) contains exactly $8=2^{3}$ nonzero entries since every column in $\left(\begin{array}{c}-\mathbf{1}_{d-1}^{\top} \\ I_{d-1}\end{array}\right)$ has exactly 2 nonzero entries.

(c) According to [22, Thm. 4] the rank of the Kronecker product of two matrices is the product of the ranks of the matrices and thus

$$
\operatorname{rank}(N)=\operatorname{rank}\left(\begin{array}{c}
-\mathbf{1}_{d-1}^{\top} \\
I_{d-1}
\end{array}\right) \operatorname{rank}\left(\begin{array}{c}
-\mathbf{1}_{d-1}^{\top} \\
I_{d-1}
\end{array}\right) \operatorname{rank}\left(\begin{array}{c}
-\mathbf{1}_{d-1}^{\top} \\
I_{d-1}
\end{array}\right)=(d-1)^{3} .
$$

(c) Rewrite

$$
A=\left(\begin{array}{c|ccc}
\mathbf{1}_{d}^{\top} \otimes I_{d} \otimes I_{d} \\
I_{d} \otimes \mathbf{1}_{d}^{\top} \otimes I_{d} \\
I_{d} \otimes I_{d} \otimes \mathbf{1}_{d}^{\top}
\end{array}\right)=\left(\begin{array}{cccc}
I_{d^{2}} & I_{d^{2}} & \cdots & I_{d^{2}} \\
\hline e_{d}^{1} \otimes \mathbf{1}_{d}^{\top} \otimes I_{d} & e_{d}^{2} \otimes \mathbf{1}_{d}^{\top} \otimes I_{d} & \cdots & e_{d}^{d} \otimes \mathbf{1}_{d}^{\top} \otimes I_{d} \\
e_{d}^{1} \otimes I_{d} \otimes \mathbf{1}_{d}^{\top} & e_{d}^{2} \otimes I_{d} \otimes \mathbf{1}_{d}^{\top} & \cdots & e_{d}^{d} \otimes I_{d} \otimes \mathbf{1}_{d}^{\top}
\end{array}\right),
$$

where $e_{d}^{i}$ denote the canonical basis vectors in $\mathbb{R}^{d}$. Define

$$
L=\left(\begin{array}{c|cc}
I_{d^{2}} & 0 & 0 \\
\hline-e_{d}^{1} \otimes \mathbf{1}_{d}^{\top} \otimes I_{d} & I_{d^{2}} & 0 \\
-e_{d}^{1} \otimes I_{d} \otimes 1_{d}^{\top} & 0 & I_{d^{2}}
\end{array}\right)
$$


and compute the product

$$
\begin{aligned}
& L A=\left(\begin{array}{c|ccc}
I_{d^{2}} & I_{d^{2}} & \cdots & I_{d^{2}} \\
\hline 0 & -e_{d}^{1} \otimes \mathbf{1}_{d}^{\top} \otimes I_{d}+e_{d}^{2} \otimes \mathbf{1}_{d}^{\top} \otimes I_{d} & \cdots & -e_{d}^{1} \otimes \mathbf{1}_{d}^{\top} \otimes I_{d}+e_{d}^{d} \otimes \mathbf{1}_{d}^{\top} \otimes I_{d} \\
0 & -e_{d}^{1} \otimes I_{d} \otimes \mathbf{1}_{d}^{\top}+e_{d}^{2} \otimes I_{d} \otimes \mathbf{1}_{d}^{\top} & \cdots & -e_{d}^{1} \otimes I_{d} \otimes \mathbf{1}_{d}^{\top}+e_{d}^{d} \otimes I_{d} \otimes \mathbf{1}_{d}^{\top}
\end{array}\right) \\
& =\left(\begin{array}{c|ccc}
I_{d^{2}} & I_{d^{2}} & \cdots & \multicolumn{1}{c}{I_{d^{2}}} \\
\hline 0 & \left(-e_{d}^{1}+e_{d}^{2}\right) \otimes \mathbf{1}_{d}^{1} \otimes I_{d} & \cdots & \left(-e_{d}^{1}+e_{d}^{d}\right) \otimes \mathbf{1}_{d}^{\top} \otimes I_{d} \\
0 & \left(-e_{d}^{1}+e_{d}^{2}\right) \otimes I_{d} \otimes \mathbf{1}_{d}^{\top} & \cdots & \left(-e_{d}^{1}+e_{d}^{d}\right) \otimes I_{d} \otimes \mathbf{1}_{d}^{\top}
\end{array}\right)
\end{aligned}
$$

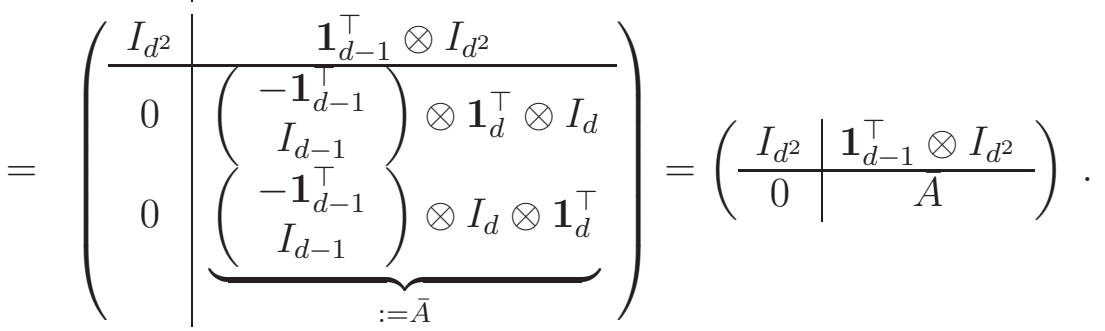

Since $L$ is a regular matrix

$$
\operatorname{rank}(A)=\operatorname{rank}(L A)=\operatorname{rank}\left(I_{d^{2}}\right)+\operatorname{rank}(\bar{A})=d^{2}+\operatorname{rank}(\bar{A}) .
$$

We further investigate the rank of $\bar{A}$. By dropping the first and $(d+1)$-th row in $\bar{A}$ we obtain a new matrix

$$
\tilde{A}:=\left(\frac{I_{d-1} \otimes \mathbf{1}_{d}^{\top} \otimes I_{d}}{I_{d-1} \otimes I_{d} \otimes \mathbf{1}_{d}^{\top}}\right)
$$

whereas

$$
\operatorname{rank}(\bar{A})=\operatorname{rank}(\tilde{A})
$$

holds. It is well known that

$$
\operatorname{rank}(\tilde{A})=\operatorname{rank}\left(\tilde{A} \tilde{A}^{\top}\right)
$$

Further, we compute

$$
G:=\left(\begin{array}{c|c|c}
d I_{d-1} \otimes I_{d} & I_{d-1} \otimes \mathbf{1}_{d}^{\top} \otimes \mathbf{1}_{d} \\
\hline I_{d-1} \otimes \mathbf{1}_{d} \otimes \mathbf{1}_{d}^{\top} & d I_{d-1} \otimes I_{d}
\end{array}\right)=\left(\begin{array}{cc}
d I_{d(d-1)} & I_{d-1} \otimes\left(\mathbf{1}_{d} \mathbf{1}_{d}^{\top}\right) \\
\hline I_{d-1} \otimes\left(\mathbf{1}_{d} \mathbf{1}_{d}^{\top}\right) & d I_{d(d-1)}
\end{array}\right) .
$$

By analogy to $L$ we define

$$
\tilde{L}:=\left(\begin{array}{c|c}
I_{d(d-1)} & 0 \\
\hline-\frac{1}{d} I_{d-1} \otimes\left(\mathbf{1}_{d} \mathbf{1}_{d}^{\top}\right) & I_{d(d-1)}
\end{array}\right),
$$

as the product of the first $d(d-1)$ Frobenius matrices respective to $G$ and compute

$$
\begin{aligned}
\tilde{L} G & =\left(\begin{array}{c|c}
d I_{d(d-1)} & I_{d-1} \otimes\left(\mathbf{1}_{d} \mathbf{1}_{d}^{\top}\right) \\
\hline-\frac{1}{d} d I_{d-1} \otimes\left(\mathbf{1}_{d} \mathbf{1}_{d}^{(}\right)+I_{d-1} \otimes\left(\mathbf{1}_{d} \mathbf{1}_{d}^{\top}\right) & -\frac{1}{d} I_{d-1} \otimes\left(\mathbf{1}_{d} \mathbf{1}_{d}^{\top}\right)^{2}+d I_{d(d-1)}
\end{array}\right) \\
& =\left(\begin{array}{c|c}
d I_{d(d-1)} & I_{d-1} \otimes\left(\mathbf{1}_{d} \mathbf{1}_{d}^{\top}\right) \\
\hline 0 & I_{d-1} \otimes \underbrace{\left(-\frac{1}{d}\left(\mathbf{1}_{d} \mathbf{1}_{d}^{\top}\right)^{2}+d I_{d}\right)}_{:=\tilde{\tilde{A}}}
\end{array}\right)
\end{aligned}
$$


In order to determine the rank of $\tilde{\tilde{A}}$, we first note that there exists a orthonormal matrix $Q \in$ $\mathbb{R}^{d \times d}$ such that

$$
\left(\mathbf{1}_{d} \mathbf{1}_{d}^{\top}\right)=Q\left(\begin{array}{cccc}
d & 0 & \cdots & 0 \\
0 & 0 & \cdots & 0 \\
\vdots & \vdots & \ddots & \vdots \\
0 & 0 & \cdots & 0
\end{array}\right) Q^{\top}
$$

Hence

$$
\begin{aligned}
\tilde{\tilde{A}} & =-\frac{1}{d}\left(\mathbf{1}_{d} \mathbf{1}_{d}^{\top}\right)^{2}+d I_{d}=-\frac{1}{d} Q\left(\begin{array}{cccc}
d & 0 & \cdots & 0 \\
0 & 0 & \cdots & 0 \\
\vdots & \vdots & \ddots & \vdots \\
0 & 0 & \cdots & 0
\end{array}\right) \underbrace{Q^{\top} Q}_{I_{d}}\left(\begin{array}{cccc}
d & 0 & \cdots & 0 \\
0 & 0 & \cdots & 0 \\
\vdots & \vdots & \ddots & \vdots \\
0 & 0 & \cdots & 0
\end{array}\right) Q^{\top}+d I_{d} \\
& =-\frac{1}{d} Q\left(\begin{array}{cccc}
d^{2} & 0 & \cdots & 0 \\
0 & 0 & \cdots & 0 \\
\vdots & \vdots & \ddots & \vdots \\
0 & 0 & \cdots & 0
\end{array}\right) Q^{\top}+Q\left(\begin{array}{cccc}
d & 0 & \cdots & 0 \\
0 & d & \cdots & 0 \\
\vdots & \vdots & \ddots & \vdots \\
0 & 0 & \cdots & d
\end{array}\right) Q^{\top} \\
& =-\frac{1}{d} Q\left(\begin{array}{cccc}
0 & 0 & \cdots & 0 \\
0 & -d & \cdots & 0 \\
\vdots & \vdots & \ddots & \vdots \\
0 & 0 & \cdots & -d
\end{array}\right) Q^{\top}
\end{aligned}
$$

and $\operatorname{rank} \tilde{\tilde{A}}=d-1$ holds. Combining this with (29) and (30) we obtain

$$
\operatorname{rank}(\tilde{A})=\operatorname{rank}\left(I_{d(d-1)}\right)+\operatorname{rank}(\tilde{\tilde{A}})=d(d-1)+(d-1)^{2} .
$$

From (27), (28) and (31) we finally get

$$
\operatorname{rank}(A)=d^{2}+d(d-1)+(d-1)^{2}=3 d^{2}-3 d+1 .
$$

(e) Using the dimension formula together with (d) we obtain

$$
\operatorname{dim}(\operatorname{ker}(A))=d^{3}-\operatorname{rank}(A)=d^{3}-3 d^{2}+3 d-1=(d-1)^{3} .
$$

Now (a) implies (e).

(f) Follows directly from (e) and the definition (9) of $N$. 


\section{Proof of Proposition 3.2}

Proof. In view of Prop. 2.2 (b), there are 8 columns in $A$ which are linearly independent. Thus $\operatorname{spark}(A) \leq 8$.

We index each entry of the matrix $A$ in (4) by two triples

$$
A_{\left(i_{k}, j_{k}, l_{k}\right),(p, q, r)}
$$

where all indices range over $\{0,1, \ldots, d-1\}$ except for $i_{1}=j_{2}=l_{3}=0$. The first triplets index rows (projection rays) corresponding to the three matrices (projection directions) stacked together, for $k=1,2,3$. The second triplets index columns (voxels).

For a fixed column, we read off from (4) the three non-zero entries

$$
\delta_{j_{1}, q} \delta_{l_{1}, r}, \quad \delta_{i_{1}, p} \delta_{l_{1}, r}, \quad \delta_{i_{1}, p} \delta_{j_{1}, q}
$$

Consequently, pairs of indices $(q, r),(p, r),(p, q)$ represent nonvanishing entries of columns $(p, q, r)$, and we represent each column by the triangle $K_{3}$ having $p, q, r$ as vertices - see Fig. 12a. The intersection of edges sets corresponds to the common support of column vectors. We show that at least 8 columns are necessary so as to have no edge that does not intersect with any other edge.

Consider two columns (triangles). They must differ in at least a single vertex $p, q$ or $r$, hence in at least two edges. Assume $(p, q)$ is the common edge. Then the two remaining vertices are $r, r^{\prime}$ with $r^{\prime} \neq r$ which cannot form the vertices of a third triangle (only triplets $(p, q, r)$ do). We conclude that any third triangle adjoined cannot share more than a single common edge, and that four triangles with maximally intersecting edge sets are arranged as shown in Fig. 12b.

The four non-intersecting edges in Fig. 12b generate non-vanishing entries in any linear combination of four columns. Because all of them have the form $\left(p^{\prime}, q^{\prime \prime}\right)$, the same reasoning as above shows that no two of them can be edges of another triangle. Hence four other triangles are needed to cover their support. We generate them with a single additional vertex - Fig. 12c - in order to combine them to a minimal dependent set - Fig. 12d. 


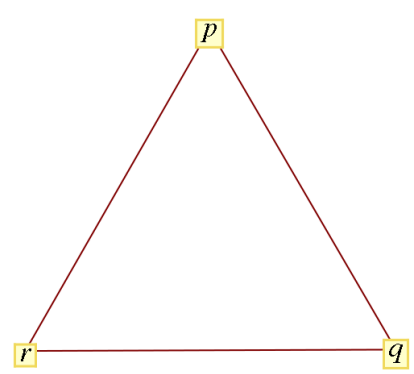

(a)

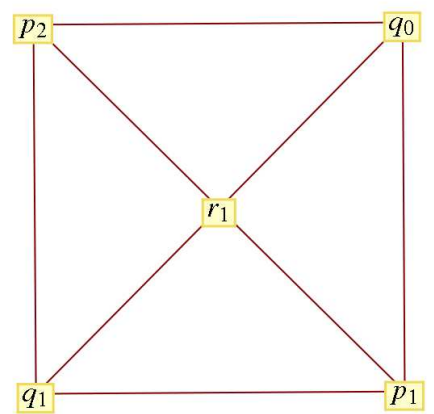

(c)

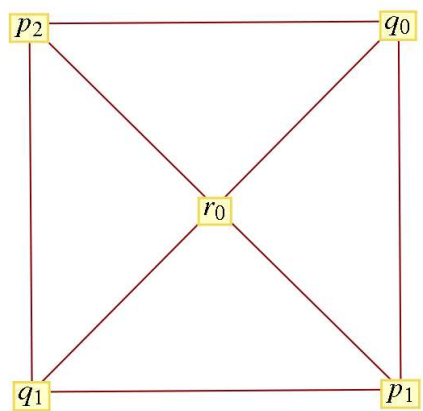

(b)

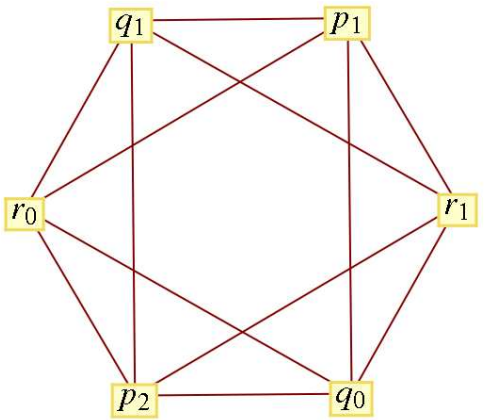

(d)

Figure 12: (a) The triangle $K_{3}$ representing a column of the matrix $A$ indexed by $(p, q, r)$. Edges represent the three non-vanishing entries. (b), (c) Two minimal configurations of 4 columns combined in (d) give a minimal dependent set. 


\section{References}

[1] Parallel Optimization: Theory, Algorithms and Applications. Oxford University Press, New York, 1997.

[2] M. Aigner. Diskrete Mathematik. Vieweg, Braunschweig/Wiesbaden, Germany, 1993.

[3] R. Baraniuk, M. Davenport, R. DeVore, and M. Wakin. A simple proof of the restricted isometry property for random matrices. Constr. Approx, 28:253-263, 2008.

[4] R. Berinde, A.C. Gilbert, P. Indyk, H. Karloff, and M.J. Strauss. Combining geometry and combinatorics: A unified approach to sparse signal recovery. In Proc. 46th Ann. Allerton Conf. Communication, Control, and Computing, pages 798-805, 2008.

[5] R. Berinde and P. Indyk. Sparse recovery using sparse random matrices. 2008. Preprint.

[6] A. Björner. Some matroid inequalities. Discrete Mathematics, 31:101-103, 1980.

[7] A.M. Bruckstein, M. Elad, and M. Zibulevsky. On the uniqueness of nonnegative sparse solutions to underdetermined systems of equations. IEEE Transactions on Information Theory, 54:4813-4820, 2008. Preprint.

[8] E. Candès. The restricted isometry property and its implications for compressed sensing. Compte Rendus de l'Academie des Sciences, Paris, Series I, 346:589-592, 2008.

[9] E. Candès, J. Romberg, and Tao T. Robust uncertainty principles: Exact signal reconstruction from highly incomplete frequency information. IEEE Trans on Information Theory, 52(2):489-509, 2006.

[10] E. Candes, M. Rudelson, T. Tao, and R. Vershynin. Error correcting via linear programming. In 46th Ann. IEEE Symp. Found. Computer Science (FOCS'05), pages 295-308, 2005 .

[11] E. Candès and Tao T. Near optimal signal recovery from random projections: Universal encoding strategies? IEEE Trans on Information Theory, 52(12):5406-5425, 2006.

[12] V. Chandar. A negative result concerning explicit matrices with the restricted isometry property. 2008. Preprint.

[13] A. Cohen, W. Dahmen, and R. DeVore. Compressed sensing and best $k$-term approximation. J. Amer. Math. Soc., 22:211-231, 2009.

[14] R.A. DeVore. Deterministic constructions of compressed sensing matrices. J. Complexity, 23:918-925, 2007.

[15] D. Donoho and J. Tanner. Neighborliness of randomly-projected simplices in high dimensions. Proceedings of the National Academy of Sciences, 102:9452-9457, 2005. 
[16] D. Donoho and J. Tanner. Thresholds for the recovery of sparse solutions via 11 minimization. In Proc. 40th Ann. Conf. on Information Sciences and Systems, pages 202-206, 2006.

[17] D. L. Donoho. Compressed sensing. IEEE Trans. Inform. Theory, 52(4):1289-1306, 2006.

[18] D.L. Donoho and M. Elad. Optimally sparse representation in general (non-orthogonal) dictionaries via $\ell_{1}$ minimization. 100:2197-2202, 2003.

[19] D.L. Donoho and J. Tanner. Sparse nonnegative solution of underdetermined linear equations by linear programming. Proc. National Academy of Sciences, 102(27):94469451, 2005.

[20] M. Elad. Journal on Applied Signal Processing, Vol. 2006:1-12, 2006.

[21] Elsinga G., Scarano F., Wieneke B., and B. van Oudheusden. Tomographic particle image velocimetry. Exp. Fluids, 41:933-947, 2007.

[22] A. Graham. Kronecker Products and Matrix Calculus With Applications. Halsted Press, John. Wiley and Sons, NY., 1981.

[23] B. Grünbaum. Convex Polytopes. Springer, 2nd. ed. prepared by kaibel, v. and klee, v. and ziegler, g. edition, 2003.

[24] P. Indyk. Explicit constructions for compressed sensing of sparse signals, booktitle =.

[25] K. Natarajan. Sparse approximate solutions to linear systems. SIAM J. Comput., 24:227234, 1995.

[26] A.M. Pinkus. On L $L_{1}$-Approximation, volume 93 of Cambridge Tracts in Math. Cambridge Univ. Press, 1989.

[27] Gordon R., Bender R., and Herman G.T. Algebraic reconstruction techniques (art) for three-dimensional electron microscopy and x-ray photography. J. Theor. Biol, 29:471$481,1970$.

[28] M. Rudelson and R. Vershynin. Geometric approach to error correcting codes and reconstruction of signals. Int. Mathematical Research Notices, 64:4019 - 4041, 2005.

[29] Petra S., Schnörr C., Schröder A., and Wieneke B. Tomographic image reconstruction in experimental fluid dynamics: Synopsis and problems. In In: Mathematical Modelling of Environmental and Life Sciences Problems. Ed Acad Romane, Bucuresti, 2007.

[30] T. Strohmer and R.W. Heath. Grassmannian frames with applications to coding and communication. Appl. Comp. Harmonic Analysis, 14(3):257-275, May 2003.

[31] Y. Zhang. A simple proof for recoverability of 11-minimization: Go over or under? 2005. Preprint. 
[32] Y. Zhang. A simple proof for recoverability of 11-minimization (ii): the nonnegativity case. 2005. Preprint. 